\title{
Efficient Implementation of Adaptive Order Reconstructions
}

\author{
M. Semplice ${ }^{1}(1) \cdot G$. Visconti ${ }^{2}$ (]) \\ Received: 8 October 2019 / Revised: 27 January 2020 / Accepted: 3 February 2020 \\ (c) Springer Science+Business Media, LLC, part of Springer Nature 2020
}

\begin{abstract}
Including polynomials with small degree and stencil when designing very high order reconstructions is surely beneficial for their non oscillatory properties, but may bring loss of accuracy on smooth data unless special care is exerted. In this paper we address this issue with a new Central WENOZ (CWENOZ) approach, in which the reconstruction polynomial is computed from a single set of non linear weights, but the linear weights of the polynomials with very low degree (compared to the final desired accuracy) are infinitesimal with respect to the grid size. After proving general results that guide the choice of the CWENOZ parameters, we study a concrete example of a reconstruction that blends polynomials of degree six, four and two, mimicking already published Adaptive Order WENO reconstructions (Arbogast et al. in SIAM J Numer Anal 56(3):1818-1947, 2018),(Balsara et al. in J Comput Phys 326:780-804, 2016). The novel reconstruction yields similar accuracy and oscillations with respect to the previous ones, but saves up to $20 \%$ computational time since it does not rely on a hierarchic approach and thus does not compute multiple sets of nonlinear weights in each cell.
\end{abstract}

Keywords CWENOZ-AO - Polynomial reconstruction · Weighted essentially nonoscillatory $\cdot$ CWENOZ · Adaptive order WENO $\cdot$ Finite volume schemes $\cdot$ Hyperbolic systems $\cdot$ Conservation and balance laws

Mathematics Subject Classification 65D05 $\cdot 65 \mathrm{M} 08 \cdot 65 \mathrm{M} 12 \cdot 76 \mathrm{M} 12$

This work was supported by the Deutsche Forschungsgemeinschaft (DFG, German Research Foundation) under Germany's Excellence Strategy_EXC-2023 Internet of Production-390621612 and by INDAM GNCS-2019 grant “Approssimazione numerica di problemi di natura iperbolica ed applicazioni”.

$凶 \quad$ M. Semplice

matteo.semplice@uninsubria.it

G. Visconti

visconti@igpm.rwth-aachen.de

1 Dipartimento di Scienza e Alta Tecnologia, Università dell'Insubria, Via Valleggio, 11, Como, Italy

2 Institut für Geometrie und Praktische Mathematik, RWTH Aachen University,

Templergraben 55, Aachen, Germany 


\section{Introduction}

This paper presents a novel, non hierarchic, construction that yields very high order essentially non oscillatory reconstructions that are useful in high order numerical schemes for conservation laws.

Let us consider the balance law $\partial_{t} u+\partial_{x} f(u)=s(u)$ and discretize the domain in cells $\Omega_{i}$ for $i=1, \ldots, N$. Following the method of lines, we introduce the cell averages $\bar{u}_{i}(t)=\int_{\Omega_{i}} u(t, x) \mathrm{d} x$ and compute their approximations $\bar{U}_{i}(t)$ by numerically integrating the system of ordinary differential equations

$$
\left\{\begin{array}{l}
\frac{\mathrm{d}}{\mathrm{d} t} \bar{U}_{i}(t)=-\frac{1}{\left|\Omega_{i}\right|}\left[\mathcal{F}_{i+1 / 2}(t)-\mathcal{F}_{i-1 / 2}(t)\right]+\mathcal{S}_{i}(t) \\
\bar{U}_{i}(0)=\bar{u}_{i}(0)
\end{array}\right.
$$

Both the numerical fluxes $\mathcal{F}_{i \pm 1 / 2}$ at the interfaces and the numerical source terms $\mathcal{S}_{i}$ are computed with the help of a reconstruction operator; this latter derives pointwise approximations $R_{i}(t, x)$, at location $x$ in the cell $i$, from the cell averages $\bar{U}_{i-l}(t), \ldots, \bar{U}_{i+r}(t)$, for some $l, r \geq 0$. For a numerical flux function $F$ compatible with $f$, we define $\mathcal{F}_{i+1 / 2}(t)=F\left(R_{i}\left(t, x_{i+1 / 2}\right), R_{i+1}\left(t, x_{i+1 / 2}\right)\right)$ and the numerical source term is computed as $\mathcal{S}_{i}(t)=\sum_{q=0}^{N_{q}} w_{q} s\left(R_{i}\left(t, x_{q}\right)\right)$, where $x_{q}$ and $w_{q}$ are the nodes and weights of a quadrature formula on $\Omega_{i}$.

The reconstruction operator should yield an accurate but non oscillatory pointwise approximation of the unknown, based on its cell averages. Beyond the second order of accuracy, one considers essentially non oscillatory reconstructions, which are typically realized by selecting (as in ENO [24]) or more commonly by blending in a nonlinear way polynomials with different degrees and/or stencils. In particular WENO, which was introduced in [41], considers a set of polynomials with equal degree but different stencils and aims at reproducing the accuracy of a higher degree interpolant when the data are locally smooth. The literature on this subject is vast and the reader may refer to [40] for a review.

The suboptimal accuracy close to critical points shown by the original design has been later overcome by new definitions of the nonlinear weights (e.g. mapped WENO[25], WENOZ[6, 11], the global average weight of [3]) or by taking the small $\epsilon$ parameter to be dependent on the local mesh size [1].

Another source of difficulty in WENO reconstructions is the possible non-existence or non-positivity of the linear weights for certain grid types and reconstruction points [39]. A quite successful proposal to address this issue was put forward by Levy, Puppo and Russo in [32] for the case of achieving a third order accurate reconstruction at cell center in one and two-dimensional uniform grids. Their CWENO3 reconstruction is a nonlinear blend of one second degree polynomial and of some first degree ones; this approach frees the linear weights from having to satisfy accuracy requirements and allows to choose them arbitrarily, independently of the reconstruction point, independently of the grid type (Cartesian/unstructured, uniform/non-uniform, conforming/non-conforming, etc). Consequently no issues regarding their existence and positivity is present. The accuracy of the CWENO3 has been studied in $[15,29]$.

The idea at the base of CWENO3, namely the use of the polynomial $P_{0}$ as in Eq. (2), has been exploited in different setups. Novel reconstructions of different orders of accuracy appeared under various names in the literature for the cases of one [3,9], two $[10,12,19,37]$ and three space dimensions $[19,31,45,46]$. Among those, $[9,10,19,37,46]$ consider non-uniform grids. Applications to stochastic Galerkin have been considered in [22] and to Hamilton-Jacobi equations in [44]. 
One of the advantages of this approach is the possibility to achieve genuinely multidimensional reconstructions that do not rely on dimensional splitting and that are, theoretically and in practice, not more challenging than the one-dimensional counterpart (see e.g. [37] for AMR grids and [18,19,46] for simplicial ones).

CWENO reconstructions of arbitrary high orders have been studied in [14] and the use of Z-weights has been pursued in $[13,16]$. A very important result of [16] is an analysis of the multidimensional oscillation indicators, leading to general results that support the design of Z-weights at arbitrary order for very general one- and multi-dimensional finite volume grids.

The optimal convergence rate on smooth data can be easily achieved when the degree gap between the central high order and the lower degree polynomials is not too high (see $[14,16])$. Thus, in the design of very high order reconstructions, one has to employ "low degree" polynomials whose stencils are still quite large and that, as a consequence, are not very good at avoiding discontinuities in complex multi-dimensional flows. For this reasons, many researchers have violated the classical hypothesis on the degree gap between high and low order polynomials when designing their reconstructions. For example, small-stencil polynomials of degree one, irrespectively of the degree of the central polynomial in $[12,19$, 47,48].

However, in order to include very low order polynomials in the pool of candidate reconstruction polynomials, special care must be exerted. A leap forward has been the proposal of the Adaptive Order WENO-AO $\left(r_{l}, \ldots, r_{2} ; r_{1}\right)$ by Balsara, Garain and Shu [5]. For $l=2$ they essentially coincide with CWENOZ reconstructions of [16], and for $l>2$ the reconstruction is a blend of the reconstruction polynomials given by WENO-AO $\left(r_{k} ; r_{1}\right)$ for $k=2, \ldots, l$. This hierarchic approach effectively enhances the stability of the reconstruction of order $r_{l}$, but reduces the accuracy suddenly to $r_{1}$ whenever a discontinuity is present the stencils of both the polynomials of level $l$ and $l-1$. Arbogast, Huang and Zhao in [2] introduced a new hierarchical construction that instead is capable of reducing gradually the accuracy from $r_{l}$ to $r_{1}$ as the discontinuity moves inward in the reconstruction stencil. WENO-AO $(7,5,3)$ and WENO-AO $(9,7,5,3)$ are considered in the numerical examples of the papers.

It should nevertheless be noted that very high order Weighted Essentially Non-Oscillatory reconstructions are quite computationally intensive. For example, profiling the code Claw1dArena ${ }^{1}$ [38] revealed that CWENO, CWENOZ and WENO-AO reconstructions of order 7 consume up to $80 \%$ of the CPU time when the method of lines and a simple numerical flux as local Lax-Friedrichs is employed. Turning on the local characteristic projection technique reduces the cost of the reconstruction to around 50-60\%, which is still quite high. This fully justifies the efforts in reducing the computational costs of very high order reconstructions. An example is given by the polynomial basis proposed in [5] that reduces the computational cost of the oscillation indicators.

In this paper we propose a novel approach to reduce the cost of adaptive order reconstructions. In Sect. 2, we revise the standard CWENO [14] and CWENOZ [16] reconstructions, as well as the WENO-AO reconstructions of [2,5]. In our approach, introduced and studied in Sect. 3, we replace the hierarchical computation of nonlinear weights of $[2,5]$ with a hierarchy of scales in the linear weights and only a set of nonlinear weights is computed. This hierarchy is realized by choosing, in a CWENOZ approach, linear weights for the very low degree polynomials that are grid-size dependent. More precisely, for a reconstruction of accuracy $G+1$, all candidate polynomials of degree smaller than $G / 2$ will have a linear weight of

1 For this test, claw1dArena was compiled with the GNU Compiler and -03 optimization level, profiling data were collected with the callgrind utility of the valgrind suite and analyzed with kcachegrind. The data reported refer to the linear advection of the Jiang-Shu profile and to the Lax shock tube with characteristic projection. 
size $\mathcal{O}\left(\Delta x^{r}\right)$ for some $r>0$. The analysis in Sect. 3 indicates how the exponent $r$ should be chosen in order to guarantee both that, on the one hand, the accuracy of the reconstruction is not reduced in case of smooth data and, on the other hand, the non-oscillatory properties of the reconstruction are boosted by the very small stencils of some candidate polynomials close to discontinuities. In Sect. 4 we report several numerical tests demonstrating that our new approach yields results that are comparable in accuracy to those obtained with the reconstructions in [2,5], but save up to $20 \%$ of computational time. Conclusions and perspectives for future work are discussed in Sect. 5.

\section{Review of CWENO Based Reconstructions}

Before introducing the new adaptive order CWENOZ reconstruction, we recall the definition of the classical CWENO reconstruction operator. Later in this section, CWENOZ and WENO-AO will be introduced, discussing their properties, but for a detailed analysis we point the reader to [13-15] for CWENO, to [13,16] for CWENOZ and to [2,5,30] for WENO-AO.

Throughout the paper we restrict the presentation to the scalar case since usually the reconstruction procedures are applied component-wise, directly to the conserved variables or after the local characteristic projection. We also concentrate on the one-dimensional setting, as multiple space dimensions may be treated either trivially by splitting or by extending the present hierarchic procedure to truly multi-dimensional CWENOZ reconstructions along the lines of $[10,19,37,46,48]$.

In order to describe a reconstruction procedure, we consider as given data the cell averages $\bar{u}_{k}$ of a function $u$ over the cells of a grid that is composed by cells $\Omega_{k} \subset \mathbb{R}$. A reconstruction aims to recover point-wise information on $u$ in the interior and at the boundaries of a cell, using the knowledge of cell averages of $u$ in that cells and its neighbors. To simplify the notation, we describe the reconstruction in a cell $\Omega_{0}$ of size size $\Delta x$ and centered at the point $x_{0}=0$.

Definition 1 Let $\mathcal{S}$ be a set of cells containing $\Omega_{0}$. The polynomial $P$ associated to the stencil $\mathcal{S}$ is the polynomial that satisfies the conservation property

$$
\frac{1}{\Delta x} \int_{\Omega_{j}} P(x) \mathrm{d} x=\bar{u}_{j}, \quad \forall \Omega_{j} \in \mathcal{S} .
$$

The interpolation property of all polynomials employed in this paper is intended in the sense of Definition 1. We point out that, especially in multi-dimensions, it is often convenient to relax the above requirement and employ least-squares fitted polynomials as in $[10,19,26$, $37,46,48$ ] and that the results of this paper, which rely only on the approximation errors of polynomials should easily extend to this more general setup.

Next we recall the definition of the Jiang-Shu oscillation indicators. Let $\mathbb{P}^{k}$ be the space of polynomials with degree at most $k \in \mathbb{N}$.

Definition 2 (see [28]) The smoothness indicator of a polynomial $P \in \mathbb{P}^{k}$ is

$$
I[P]:=\sum_{i=1}^{k} \Delta x^{2 i-1} \int_{\Omega_{0}}\left(\frac{\mathrm{d}^{i}}{\mathrm{~d} x^{i}} P(x)\right)^{2} \mathrm{~d} x .
$$

We recall that $I[P]=\mathcal{O}(1)$ even if a discontinuity is present in the stencil of $P$ and that $I[P] \rightarrow 0$ under grid refinement if $P$ is associated to smooth data. 
We point out that Definition 2 for the smoothness indicator is not adequate for classes of problems where the solution is expected to be continuous and for example for HamiltonJacobi equations the summation in (1) should start from $i=2$ in order to disregard the contribution of the $L^{2}$ norm of the first derivative [21,27]. Also, different approaches to define smoothness indicators have been explored in the literature: see e.g. [23] for a definition based on $L^{1}$ norms. Of course, in order to employ different indicators in the constructions of this paper, one would need to first prove a counterpart of Proposition 2 of Sect.3.1.1.

\subsection{CWENO Reconstructions}

We recall here the general definition of a CWENO reconstruction, which was originally given in [32] for schemes of order 3 and later generalized to arbitrary order and analyzed in [14].

Definition 3 (CWENO operator) Given a stencil $\mathcal{S}_{\text {opt }}$ of $G+1$ cells that includes $\Omega_{0}$, let $P_{\text {opt }} \in \mathbb{P}^{G}$ (optimal polynomial) be the polynomial of degree $G$ which interpolates all the given data in $\mathcal{S}_{\text {opt }}$. Further, let $P_{1}, P_{2}, \ldots, P_{m}$ be a set of $m \geq 1$ polynomials of degree $g$ with $g<G$ such that, for $k=1, \ldots, m, P_{k}$ interpolates the cell averages of a sub-stencil $\mathcal{S}_{k}$, chosen such that $\Omega_{0} \in \mathcal{S}_{k} \subset \mathcal{S}_{\text {opt }}$. Let also $\left\{d_{k}\right\}_{k=0}^{m}$ be a set of strictly positive real coefficients such that $\sum_{k=0}^{m} d_{k}=1$.

The CWENO operator computes a reconstruction polynomial

$$
P_{\text {rec }}=\operatorname{CWENO}\left(P_{\text {opt }} ; P_{1}, \ldots, P_{m}\right) \in \mathbb{P}^{G}
$$

as follows:

1. introduce the polynomial $P_{0}$ defined as

$$
P_{0}(x)=\frac{1}{d_{0}}\left(P_{\mathrm{opt}}(x)-\sum_{k=1}^{m} d_{k} P_{k}(x)\right) \in \mathbb{P}^{G} ;
$$

2. compute the regularity indicators

$$
I_{0}=I\left[P_{\mathrm{opt}}\right], \quad I_{k}=I\left[P_{k}\right], k \geq 1
$$

3. compute the nonlinear coefficients $\left\{\omega_{k}\right\}_{k=0}^{m}$ as

$$
\alpha_{k}=\frac{d_{k}}{\left(I_{k}+\epsilon\right)^{\ell}}, \quad \omega_{k}=\frac{\alpha_{k}}{\sum_{i=0}^{m} \alpha_{i}},
$$

where $\epsilon$ is a small positive quantity, and $\ell \geq 1$

4. define the reconstruction polynomial as

$$
P_{\text {rec }}(x)=\sum_{k=0}^{m} \omega_{k} P_{k}(x) \in \mathbb{P}^{G} .
$$

We point out that the use of the additional polynomial $P_{0}$ defined in Eq. (2) is what characterizes a Central WENO based reconstruction. This allows to employ linear coefficients $\left\{d_{k}\right\}_{k=0}^{m}$ which do not depend on the reconstruction point and consequently the CWENO operator defines a reconstruction polynomial which is globally defined and uniformly accurate on the computational cell. $P_{\text {rec }}$ can be later evaluated at any point, and no a-priori knowledge of reconstruction point is exploited in the computation of $P_{\text {rec }}$. This constitutes also a computational gain since the nonlinear coefficients can be computed once per cell and not once 
per reconstruction point, as in the standard WENO [40]. This makes the CWENO procedures more appealing for balance laws, multidimensional computations and unstructured meshes than their WENO counterparts. It should be noted that the reconstruction proposed in [20] also computes a single set of nonlinear weights per cell, but it requires much larger stencils than CWENO.

Next, we briefly present some results shared by all CWENO reconstructions.

Remark 1 Since all the interpolating polynomials involved in Definition 3 satisfy the conservation property on the reconstruction cell $\Omega_{0}, P_{0}$ is conservative on $\Omega_{0}$ in the sense of Definition 1 and thus also $\frac{1}{\Delta x} \int_{\Omega_{0}} P_{\text {rec }}(x) \mathrm{d} x=\bar{u}_{0}$, which shows that the CWENO operator is conservative.

The accuracy and non-oscillatory properties of CWENO based schemes are guaranteed by the dependence of their nonlinear weights on the Jiang-Shu regularity indicators. Since for any sufficiently regular function $u(x)$ the approximation orders of $P_{\mathrm{opt}} \in \mathbb{P}^{G}$ and $P_{k} \in \mathbb{P}^{g}$, for $k=1, \ldots, m$, are

$$
\left|P_{\mathrm{opt}}(x)-u(x)\right|=\mathcal{O}\left(\Delta x^{G+1}\right) \quad \text { and } \quad\left|P_{k}(x)-u(x)\right|=\mathcal{O}\left(\Delta x^{g+1}\right) \quad \forall x \in \Omega_{0},
$$

it is immediate to show that the reconstruction error at $x$ is at least of order $g+1$. Therefore, on smooth data, the nonlinear weights must be designed so that the accuracy of the reconstruction polynomial $P_{\text {rec }}$ is boosted to the accuracy of $P_{\text {opt }}$, i.e. $G+1$. More precisely, if $d_{k}-\omega_{k}$ is at least $\mathcal{O}\left(\Delta x^{G-g}\right)$ then the accuracy of the CWENO reconstruction equals the accuracy of $P_{\text {opt }}$. Provided that $G \leq 2 g$ this condition is verified if $\epsilon=\mathcal{O}\left(\Delta x^{\hat{m}}\right), \hat{m}=1,2$, see [14, Proposition 1].

On the other hand, if there were an oscillating polynomial $P_{\hat{k}}$ for some $\hat{k} \in\{1, \ldots, m\}$, then $I_{\hat{k}} \asymp 1$, i.e. $\lim _{\Delta x \rightarrow 0} I_{\hat{k}}$ exists, is finite and nonzero, and thus the corresponding nonlinear weight tends to 0 ; moreover, as proved in [14], also the nonlinear weight of $P_{0}$ would tend to 0 and the reconstruction polynomial $P_{\text {rec }}$ would become a nonlinear combination of polynomials of degree $g$ : the accuracy of the reconstruction reduces to $g+1$, but spurious oscillations in the PDE solution would be controlled.

The positive parameter $\epsilon$ prevents the division by zero in the computation of the nonlinear weights (3). In [15,29], the authors proved that the choice of $\epsilon$ can influence the convergence of the method on smooth parts of the solution. For instance, choosing $\epsilon=\mathcal{O}\left(\Delta x^{\hat{m}}\right)$, for some $\hat{m} \in \mathbb{N}$, helps to have a more regular convergence history than taking $\epsilon$ as fixed value. A convergence analysis of the scheme on smooth data gives a range of values for $\hat{m}$ that guarantees optimal order; within this range one would take $\hat{m}$ as large as possible in order to avoid spurious oscillations on discontinuous data. In the following we always consider a mesh-size dependent $\epsilon$.

In Definition 3 the number $m$ and the degree $g$ of the lower-degree polynomials is not specified nor linked to the degree $G$ of the optimal polynomial. However, the relation $G \leq$ $2 g$ is required for accuracy and in a one space dimension reconstruction of order $2 r-$ 1 it is customary to choose $g=r-1, m=g+1$ and $P_{\text {opt }}$ of degree $G=2 r-2$. This latter is determined by the exact interpolation of the data in a symmetric stencil $\mathcal{S}_{\text {opt }}$ centered on $\Omega_{0}$ containing the cells $\Omega_{-g}, \ldots, \Omega_{g}$. Furthermore, for $k=1, \ldots, m$, the lower-degree polynomials $P_{k}$ are defined as the exact interpolants on the substencils $\mathcal{S}_{k}=$ $\left\{\Omega_{k-r}, \ldots, \Omega_{k-1}\right\} \subset \mathcal{S}_{\text {opt }}$. This is the same choice considered in [13,14]. 


\subsubsection{CWENOZ}

CWENOZ was originally proposed in [13] and, recently, it was extensively analyzed in [16] in a multi-dimensional setting. We recall here its definition.

Definition 4 (CWENOZ reconstruction) Given a stencil $\mathcal{S}_{\text {opt }}$ of $G+1$ cells that includes $\Omega_{0}$, let $P_{\text {opt }} \in \mathbb{P}^{G}$ (optimal polynomial) be the polynomial of degree $G$ which interpolates all the given data in $\mathcal{S}_{\text {opt }}$. Further, let $P_{1}, P_{2}, \ldots, P_{m}$ be a set of $m \geq 1$ polynomials of degree $g$ with $g<G$ such that, for $k=1, \ldots, m, P_{k}$ interpolates the cell averages of a sub-stencil $\mathcal{S}_{k}$, chosen such that $\Omega_{0} \in \mathcal{S}_{k} \subset \mathcal{S}_{\text {opt }}$. Let also $\left\{d_{k}\right\}_{k=0}^{m}$ be a set of strictly positive real coefficients such that $\sum_{k=0}^{m} d_{k}=1$.

The CWENOZ operator

$$
P_{\text {rec }}=\operatorname{CWENOZ}\left(P_{\text {opt }} ; P_{1}, \ldots, P_{m}\right) \in \mathbb{P}^{G}
$$

is defined as the CWENO operator of Definition 3, but replacing the definition of the nonlinear coefficients (3) with $\left\{\omega_{k}\right\}_{k=0}^{m}$ defined as

$$
\alpha_{k}=d_{k}\left(1+\left(\frac{\tau}{I_{k}+\epsilon}\right)^{\ell}\right), \quad \omega_{k}=\frac{\alpha_{k}}{\sum_{i=0}^{m} \alpha_{i}},
$$

where $\epsilon$ is a small positive quantity, $\ell \geq 1$ and $\tau$ is a global smoothness indicator. For efficiency, $\tau$ is restricted to be a linear combination of the other smoothness indicators $I_{0}=$ $I\left[P_{\mathrm{opt}}\right], \ldots, I_{m}=I\left[P_{m}\right]$, i.e.

$$
\tau:=\left|\sum_{k=0}^{m} \lambda_{k} I_{k}\right|
$$

for some choice of real coefficients $\lambda_{0}, \ldots, \lambda_{m}$.

In [16], it is argued the coefficients should satisfy $\sum_{k=0}^{m} \lambda_{k}=0$ and it is also shown how to optimize their choice.

We point out that the CWENOZ reconstruction is a CWENO based reconstruction and therefore it shares the advantages of CWENO discussed in Sect. 2.1. However, the CWENOZ differs from the classical definition of the CWENO scheme in the computation of the nonlinear coefficients (5): the CWENOZ method uses the idea of Borges, Carmona, Costa and Don in [6], where they introduced in a WENO setting Definition (5) of the nonlinear coefficients, which drives them closer to their optimal values in the smooth case.

The definition of the new nonlinear weights guarantees a weaker condition on $\hat{m}$ to be satisfied in order to reach optimal accuracy, which implies the possibility to employ a smaller $\epsilon$ than in the corresponding CWENO reconstruction, enhancing their non-oscillatory properties. In [16] a thorough analysis of the global smoothness indicator is performed and the optimal definition of the nonlinear weights in multi-dimensional CWENOZ reconstructions is given. In particular, for conditions on $\ell$ and $\hat{m}$ we refer to [16, Theorem 24].

\subsubsection{WENO-AO}

Adaptive order reconstructions based on the CWENO procedure have been considered in recent years. We focus on the WENO-AO schemes and the formulations given in [5] and [2], recasting them as hierarchic CWENOZ reconstructions. In particular, we focus on the 
WENO-AO scheme of order 7 as given in [5] since it will be later used for comparisons with our 7-th order adaptive reconstruction introduced in Sect. 3.2.

Let $\mathcal{S}_{\text {opt }}$ be a stencil of seven cells centered on the computational cell $\Omega_{0}$. The WENO-AO $(7,5,3)$ reconstruction may be described as a nonlinear blending of CWENOZ reconstructions and, using the notation of this work, it can be thus formulated as

$$
\begin{aligned}
& P_{\text {rec }}=\operatorname{CWENOZ}\left(P_{\text {rec }}^{(7)} ; P_{\text {rec }}^{(5)}\right), \text { where } \\
& P_{\text {rec }}^{(7)}=\operatorname{CWENOZ}\left(P^{(7)} ; P_{L}^{(3)}, P_{C}^{(3)}, P_{R}^{(3)}\right), \\
& P_{\text {rec }}^{(5)}=\operatorname{CWENOZ}\left(P^{(5)} ; P_{L}^{(3)}, P_{C}^{(3)}, P_{R}^{(3)}\right)
\end{aligned}
$$

and $P^{(7)}$ is the 6-th degree polynomial interpolating the data in $\mathcal{S}_{\mathrm{opt}}, P^{(5)}$ is the 4-th degree polynomial interpolating the data in $\left\{\Omega_{-2}, \ldots, \Omega_{2}\right\}$ and, finally, $P_{L}^{(3)}, P_{C}^{(3)}, P_{R}^{(3)}$ are the parabolas interpolating the data of the substencils $\left\{\Omega_{k-3}, \Omega_{k-2}, \Omega_{k-1}\right\}$ for $k=1,2,3$, respectively.

As consequence of Definition (7), we have that the reconstruction polynomial $P_{\text {rec }}$ has the classical property of being globally defined on the computational cell $\Omega_{0}$ and being there uniformly accurate. However, it is clear that in order to compute $P_{\text {rec }}$ we need to proceed through three reconstruction steps. First, two inner CWENOZ procedures are applied in order to define two reconstruction polynomials of order 7 and 5 respectively. Finally, $P_{\text {rec }}$ is computed with a CWENOZ operator having inputs $P_{\text {rec }}^{(7)}$ and $P_{\text {rec }}^{(5)}$. For a detailed description of the linear and nonlinear weights employed in the WENO-AO reconstruction we refer to [5]. This procedure requires the computation of three sets of nonlinear weights and two intermediate polynomials, which makes this approach computationally expensive, especially in view of generalizations to higher orders and thus deeper hierarchies.

A more general WENO-AO reconstruction has been proposed in [2] which has no base levels and, in addition, the definition has been slightly modified leading to a gain in the computational cost. In fact, the corresponding scheme of order 7 requires the computation of only two sets of nonlinear weights instead of three.

Further, we recall that WENO-AO reconstructions have been recently designed for unstructured meshes [4] and for $\mathrm{P}_{\mathrm{N}} \mathrm{P}_{\mathrm{M}}$ schemes [7].

In the numerical tests of this paper we will refer to the WENO-AO $(7,5,3)$ reconstruction of [2] with WAO-AHZ and with WAO-BGS to the one of [5], but in its finite volume formulation as given in [2].

\section{New Adaptive Order CWENOZ Reconstruction}

Both WENO-AO reconstructions [2,5] share the following feature: more than one set of nonlinear weights must be computed for each computational cell. Our goal is to introduce an adaptive order reconstruction in which multiple computation of nonlinear weights are not needed, with the aim of saving computational time.

\subsection{Definition and Accuracy Analysis}

The definition of the new method relies on the following generalization of CWENOZ and as such it belongs to the class of CWENO reconstructions. The hierarchy of nested CWENOZ 
operators of $[2,5]$ is replaced by a hierarchy in the size of the linear weights, generalizing the approach of [33].

Definition 5 (CWENOZ-AO reconstruction.) Given a stencil $\mathcal{S}_{\text {opt }}$ of $G+1$ cells that includes $\Omega_{0}$, let $P_{\text {opt }} \in \mathbb{P}^{G}$ (optimal polynomial) be the polynomial of degree $G$ associated to $\mathcal{S}_{\text {opt }}$. Let $P_{1}, P_{2}, \ldots, P_{m}$ be a set of $m \geq 1$ polynomials of degree $g$ with $G / 2 \leq g<G$ such that, for $k=1, \ldots, m, P_{k}$ is associated to a substencil $\mathcal{S}_{k}$ with $\Omega_{0} \in \mathcal{S}_{k} \subset \mathcal{S}_{\text {opt }}$. Further, let $Q_{1}, Q_{2}, \ldots, Q_{n}$ be a set of $n \geq 1$ polynomials of degree $\gamma_{k}$ with $\gamma_{k}<G / 2$, for $k=1, \ldots, n$, and such that, for $k=1, \ldots, n, Q_{k}$ is associated to a substencil $\widetilde{\mathcal{S}}_{k}$ with $\Omega_{0} \in \widetilde{\mathcal{S}}_{k} \subset \mathcal{S}_{\text {opt }}$.

The adaptive order CWENOZ-AO reconstruction computes a reconstruction polynomial by means of a CWENOZ operator

$$
\begin{aligned}
P_{\text {rec }} & =\operatorname{CWENOZ-AO}\left(P_{\mathrm{opt}} ; P_{1}, \ldots, P_{m} ; Q_{1}, \ldots, Q_{n}\right) \\
& =\operatorname{CWENOZ}\left(P_{\mathrm{opt}} ; P_{1}, \ldots, P_{m}, Q_{1}, \ldots, Q_{n}\right)
\end{aligned}
$$

where the linear coefficients for the polynomials $Q_{1}, \ldots, Q_{n}$ are infinitesimal.

In particular we consider $\delta_{k}=\Delta x^{r_{k}}$ for some $r_{k}>0$, for $k=1, \ldots, n$, and a set of strictly positive real coefficients $\left\{d_{k}\right\}_{k=0}^{m}$ such that $\sum_{k=0}^{m} d_{k}+\sum_{k=1}^{n} \delta_{k}=1$.

The reconstruction is computed as follows:

$$
P_{\text {rec }}(x)=\sum_{k=0}^{m} \omega_{k}^{P} P_{k}(x)+\sum_{k=1}^{n} \omega_{k}^{Q} Q_{k}(x) \in \mathbb{P}^{G},
$$

where $\omega_{k}^{P}$ and $\omega_{k}^{Q}$ are the nonlinear weights computed as in Eq. (5) for the polynomial $P_{k}$ and for the polynomial $Q_{k}$, respectively. We employ a different notation for the two subsets of nonlinear weights only for convenience in the proofs. However, the only difference between $\omega_{k}^{P}$ and $\omega_{k}^{Q}$ is the fact that $\omega_{k}^{P}$ results from (5) with a linear weight $d_{k}=\mathcal{O}(1)$, while $\omega_{k}^{Q}$ results from (5) but has an infinitesimal linear weight $\delta_{k}=o(1)$.

The additional polynomial $P_{0}$ is defined as

$$
P_{0}(x)=\frac{1}{d_{0}}\left(P_{\mathrm{opt}}(x)-\sum_{k=1}^{m} d_{k} P_{k}(x)-\sum_{k=1}^{n} \delta_{k} Q_{k}(x)\right) \in \mathbb{P}^{G} .
$$

The global smoothness indicator $\tau$ is given as a linear combination of $I_{0}, \ldots, I_{m}^{P}$ :

$$
\tau:=\left|\sum_{k=0}^{m} \lambda_{k} I_{k}^{P}\right|
$$

for some choice of coefficients $\lambda_{0}, \ldots, \lambda_{m}$ such that $\sum_{k=0}^{m} \lambda_{k}=0$.

We point out that the role of $\tau$ is to detect smoothness in the large stencil of $P_{\text {opt }}$. In view of this we did not find useful to include any of the indicators $I_{k}^{Q}$ in its definition.

Notice that in Definition 5 we already fix the relation between $G$ and $g$ as $G \geq 2 g$. In addition, and compared to the CWENOZ reconstruction, the new procedure allows the use of polynomials $Q_{k}$ having degree $\gamma_{k}<g$. Classical schemes are characterized by the fact that for very large $G$, the stencil of the polynomials of degree $g$ are still quite large and it may be difficult to avoid discontinuities, especially in multi-dimension. Therefore, using lower degree polynomials helps to select smooth stencils but it may influence the optimal accuracy of the method on smooth data. However the fact that the linear weights $\delta_{k}$ associated to the low order polynomials $Q_{k}$ are $\delta_{k}=o(1)$, allows to reach accuracy on smooth data without relying on hierarchic nonlinear blending of CWENO operators, which requires multiple computations of the nonlinear weights as in the WENO-AO and similar reconstructions [2,5]. 


\subsubsection{Accuracy Analysis on Smooth Solutions}

We point out that the polynomial $P_{0}$ appearing in Definition 5 has degree $G$, but its accuracy may be degraded down to $\min _{k} \gamma_{k}<g$. However, choosing $r_{k} \geq g-\gamma_{k}$ ensures that the accuracy of $P_{0}$ is unaffected by the presence of the polynomials $Q_{1}, \ldots, Q_{m}$ in Definition 5, as detailed in the following result.

Lemma 1 Let $P_{\text {opt }}, P_{k}, k=1, \ldots, m$, and $Q_{k}, k=1, \ldots, n$, as in Definition 5. Let $u$ be a sufficiently smooth function. The polynomial $P_{0}$ in $E q$. (9) is of degree $G$, but its accuracy order in the computational cell $\Omega_{0}$ is $g$, provided that $r_{k} \geq g-\gamma_{k}$.

Proof After the Definition (9) of $P_{0}$, for each $x$ in the computational cell $\Omega_{0}$ we have

$$
\begin{aligned}
P_{0}(x)-u(x) & =\frac{1}{d_{0}}\left[P_{\mathrm{opt}}(x)-\sum_{k=1}^{m} d_{k} P_{k}(x)-\sum_{k=1}^{n} \delta_{k} Q_{k}(x)-d_{0} u(x)\right] \\
& =\frac{1}{d_{0}}\left[P_{\mathrm{opt}}(x)-\sum_{k=1}^{m} d_{k} P_{k}(x)-\sum_{k=1}^{n} \delta_{k} Q_{k}(x)-\left(1-\sum_{k=1}^{m} d_{k}-\sum_{k=1}^{n} \delta_{k}\right) u(x)\right] \\
& =\frac{1}{d_{0}} \underbrace{\left(P_{\mathrm{opt}}(x)-u(x)\right)}_{\mathcal{O}\left(\Delta x^{G+1}\right)}+\frac{1}{d_{0}} \sum_{k=1}^{n} d_{k} \underbrace{\left(u(x)-P_{k}(x)\right)}_{\mathcal{O}\left(\Delta x^{g+1}\right)}+\frac{1}{d_{0}} \sum_{k=1}^{m} \delta_{k} \underbrace{\left(u(x)-Q_{k}(x)\right)}_{\mathcal{O}\left(\Delta x^{\gamma_{k}+1}\right)} .
\end{aligned}
$$

Therefore, $P_{0}(x)-u(x)=\mathcal{O}\left(\Delta x^{g}\right)$ if $\delta_{k}=\mathcal{O}\left(\Delta x^{r_{k}}\right)$ with $r_{k} \geq g-\gamma_{k}$.

We can now state sufficient conditions to ensure that, for smooth data, the reconstruction error at each point in the computational cell of the new CWENOZ-AO scheme is not bigger than the interpolation error of $P_{\text {opt }}$.

Lemma 2 Let $P_{\text {rec }}$ be a polynomial as in Eq. (8) in Definition (5). Let be $u$ a sufficiently smooth function. Then, the approximation error of $P_{\text {rec }}$ in the computational cell $\Omega_{0}$ is of order $G+1$ under the restriction $\gamma_{k}+r_{k} \geq g$ provided that both $\frac{w_{k}^{P}-d_{k}}{d_{k}}=\mathcal{O}\left(\Delta x^{G-g}\right)$ and $\frac{w_{k}^{Q}-\delta_{k}}{\delta_{k}}=\mathcal{O}\left(\Delta x^{G-\gamma_{k}-r_{k}}\right)$ hold true.

Proof The approximation errors of the polynomials appearing in Definition 5 are

$$
\begin{aligned}
\left|P_{\mathrm{opt}}(x)-u(x)\right| & =\mathcal{O}\left(\Delta x^{G+1}\right), \quad\left|P_{k}(x)-u(x)\right|=\mathcal{O}\left(\Delta x^{g+1}\right), \\
\left|Q_{k}(x)-u(x)\right| & =\mathcal{O}\left(\Delta x^{\gamma_{k}+1}\right)
\end{aligned}
$$

at any point $x$ in the computational cell. Then the reconstruction error at $x$

$$
\begin{aligned}
u(x)-P_{\text {rec }}(x)= & \underbrace{\left(u(x)-P_{\mathrm{opt}}(x)\right)}_{O\left(\Delta x^{G+1}\right)}+\left(d_{0}-w_{0}^{P}\right) \underbrace{\left(P_{0}(x)-u(x)\right)}_{O\left(\Delta x^{g+1}\right)} \\
& +\sum_{k=1}^{m}\left(d_{k}-w_{k}^{P}\right) \underbrace{\left(P_{k}(x)-u(x)\right)}_{O\left(\Delta x^{g+1}\right)}+\sum_{k=1}^{n}\left(\delta_{k}-w_{k}^{Q}\right) \underbrace{\left(Q_{k}(x)-u(x)\right)}_{O\left(\Delta x^{\gamma_{k}+1}\right)}
\end{aligned}
$$

is of optimal order $G+1$ if $w_{k}^{P}-d_{k}=\mathcal{O}\left(\Delta x^{G-g}\right)$ and $w_{k}^{Q}-\delta_{k}=\mathcal{O}\left(\Delta x^{G-\gamma_{k}}\right)$. The first is equivalent to $\frac{w_{k}^{P}-d_{k}}{d_{k}}=O\left(\Delta x^{G-g}\right)$ since $d_{k}=\mathcal{O}(1)$. The latter is implied by $\frac{w_{k}^{Q}-\delta_{k}}{\delta_{k}}=$ $\mathcal{O}\left(\Delta x^{G-\gamma_{k}-r_{k}}\right)$. 
The sufficient conditions of the previous result clarify the roles played by the nonlinear weights and by the linear weights in ensuring the optimal convergence rates on smooth data. The computation of the nonlinear weights should ensure that, for all polynomials, they are close to their linear counterpart in a relative sense, as $\mathcal{O}\left(\Delta x^{G-g}\right)$, independently of $\gamma_{k}$. On the other hand, the linear weights $\delta_{k}=\Delta x^{r_{k}}$ make up for the difference $g-\gamma_{k}$.

For the analysis, let us assume that the cell averages to which the reconstruction is applied are sampled exactly from a sufficiently smooth function $u(x)$ that may have a critical point of order $n_{c p} \geq 0$ at a point $x_{0} \in \Omega_{0}$. Obviously $n_{c p}=0$ means that there is no critical point.

Let us first prove a very general feature of the smoothness indicators.

Proposition 1 Let $\mathcal{S}$ be a stencil including $\Omega_{0}$ and let $q(x)$ be a polynomial approximating a regular function $u(x)$. Then

$$
I[q]=\mathcal{O}\left(\Delta x^{2 n_{c p}+2}\right) .
$$

Proof Since the regularity indicators are invariant if data are shifted by an additive constant, without loss of generality we assume that $u\left(x_{0}\right)=0$. In the following, we will consider the Taylor expansion of $u(x)$ around $x_{0}$ and denote for simplicity $u_{0}^{(k)}=u^{(k)}\left(x_{0}\right)$. The cell average of $u(x)$ in $\Omega_{\alpha}$ will have an expansion of the form

$$
\bar{u}_{\alpha}=\sum_{k \geq n_{c p}+1} A_{k}^{\alpha} u_{0}^{(k)} \Delta x^{k}
$$

for some constants $A_{k}^{\alpha} \in \mathbb{R}$. The regularity indicator is quadratic with respect to the data (see [16, Proposition 13]), and thus there exists $C_{\alpha \beta} \in \mathbb{R}$ such that

$$
\begin{aligned}
I[q] & =\sum_{\left\{\alpha, \beta: \Omega_{\alpha}, \Omega_{\beta} \in \mathcal{S}\right\}} C_{\alpha \beta} \bar{u}_{\alpha} \bar{u}_{\beta} \\
& =\sum_{\left\{\alpha, \beta: \Omega_{\alpha}, \Omega_{\beta} \in \mathcal{S}\right\}} C_{\alpha \beta}\left(\sum_{k \geq n_{c p}+1} A_{k}^{\alpha} u_{0}^{(k)} \Delta x^{k}\right)\left(\sum_{j \geq n_{c p}+1} A_{j}^{\beta} u_{0}^{(j)} \Delta x^{j}\right) \\
& =\mathcal{O}\left(\Delta x^{2 n_{c p}+2}\right) .
\end{aligned}
$$
22].

Next, we recall some results proven in [16, Proposition 14, Corollary 16 and Corollary

Proposition 2 Let $\mathcal{S}$ be a stencil including $\Omega_{0}$ and let $q(x)$ be a polynomial approximating a regular function $u(x)$ with accuracy greater or equal than $M$, then

$$
I[q]=B_{M}+R[q]
$$

where $B_{M}$ depends on $M$ but not on $q(x)$, while $R[q]$ depends on the stencil $\mathcal{S}$ and $R[q]=$ $o\left(B_{M}\right)$. Moreover, if $u$ has a critical point with $n_{c p} \geq M$, then $B_{M}=0$ and $R[q]=$ $\mathcal{O}\left(\Delta x^{2 M+2}\right)$. In the case $n_{c p}<M$, then $B_{M}=\mathcal{O}\left(\Delta x^{2\left(n_{c p}+1\right)}\right)$ and $R[q]=\mathcal{O}\left(\Delta x^{M+2+n_{c p}}\right)$, so that $R[q]=o\left(B_{M}\right)$. Further, thanks to the hypothesis $\sum_{k=0}^{m} \lambda_{k}=0, \tau=\mathcal{O}\left(\sum_{k=0}^{m} R_{k}\right)$.

Lemma 3 (Lemma 6 of [17]) If $\alpha_{k}^{P}=d_{k}\left(1+A \Delta x^{\beta}+\mathcal{O}\left(\Delta x^{\beta+1}\right)\right)$ for $k=0, \ldots, m$, and $\alpha_{k}^{Q}=\delta_{k}\left(1+A \Delta x^{\beta}+\mathcal{O}\left(\Delta x^{\beta+1}\right)\right)$ for $k=1, \ldots, n$ with $\beta>0$ and $A$ independent on $k$, then $\frac{\omega_{k}^{P}}{d_{k}}=1+\mathcal{O}\left(\Delta x^{\beta+1}\right)$ for $k=0, \ldots, m$ and $\frac{\omega_{k}^{Q}}{\delta_{k}}=1+\mathcal{O}\left(\Delta x^{\beta+1}\right)$ for $k=1, \ldots, n$. 
Proof The proof relies on observing that

$$
\sum_{j=0}^{m} \alpha_{j}^{P}+\sum_{j=1}^{m} \alpha_{j}^{Q}=1+A \Delta x^{\beta}+\mathcal{O}\left(\Delta x^{\beta+1}\right) .
$$

Then

$$
\omega_{k}^{P}=d_{k}\left(1+A \Delta x^{\beta}+\mathcal{O}\left(\Delta x^{\beta+1}\right)\right)\left(1-A \Delta x^{\beta}+\mathcal{O}\left(\Delta x^{\beta+1}\right)\right)
$$

and similarly for $\omega_{k}^{Q}$.

We are now ready to analyze the behavior of the reconstruction on smooth data. In the following, given a function $q(\Delta x)$, we will write $\theta(q(\Delta x))=r$ to mean that $q(\Delta x) \sim a_{r} \Delta x^{r}$ for some $a_{r} \neq 0$.

Case (A): $\boldsymbol{n}_{\boldsymbol{c} \boldsymbol{p}} \geq \boldsymbol{g}$ Applying Proposition 2 with $M=g$ we have that $B_{g}=0$ and thus, defining $R_{k}=R\left[P_{k}\right]$, we can write for $k=0, \ldots, m$ that

$$
\alpha_{k}^{P}=d_{k}\left(1+\left(a_{k}\right)^{\ell}\right) \quad \text { with } \quad a_{k}=\frac{\tau}{I_{k}^{P}+\epsilon}=\frac{\tau}{R_{k}+\epsilon} \sim \frac{C_{\tau} \Delta x^{\theta(\tau)}}{C_{k} \Delta x^{\theta\left(R_{k}\right)}+C_{\epsilon} \Delta x^{\hat{m}}} .
$$

After Proposition 2 we have that both $\theta(\tau)$ and $\theta\left(R_{k}\right)$ are larger than $2 g+2$. Then restricting the choice of $\hat{m}$ to

$$
\hat{m} \leq 2 g+1
$$

ensures that $a_{k} \sim C \Delta x^{\theta(\tau)-\hat{m}} \rightarrow 0$ with $C=C_{\tau} / C_{\epsilon}$ independent on $k$. Similarly, for $k=1, \ldots, n$,

$$
\alpha_{k}^{Q}=\delta_{k}\left(1+\left(b_{k}\right)^{\ell}\right) \quad \text { with } \quad b_{k}=\frac{\tau}{I_{k}^{Q}+\epsilon} \sim \frac{C_{\tau} \Delta x^{\theta(\tau)}}{\tilde{C}_{k} \Delta x^{\theta\left(I_{k}^{Q}\right)}+C_{\epsilon} \Delta x^{\hat{m}}}
$$

and, using condition (11), $b_{k} \sim C \Delta x^{\theta(\tau)-\hat{m}} \rightarrow 0$ with $C=C_{\tau} / C_{\epsilon}$. This is trivial if $\gamma_{k}=0$ and otherwise it holds thanks to Proposition 1, which ensures $\theta\left(I_{k}^{Q}\right) \geq 2 g+2$.

We thus have that $\alpha_{k}^{P}=d_{k}\left(1+C^{\ell} \Delta x^{\ell t}+\mathcal{O}\left(\Delta x^{\ell t+1}\right)\right)$ for $k=0, \ldots, m$ and $\alpha_{k}^{Q}=$ $\delta_{k}\left(1+C^{\ell} \Delta x^{\ell t}+\mathcal{O}\left(\Delta x^{\ell t+1}\right)\right)$ for $k=1, \ldots, n$, where we have defined $t=\theta(\tau)-\hat{m}$. We can thus apply Lemma 3 to conclude that $\frac{\omega_{k}^{P}-d_{k}}{d_{k}}=\mathcal{O}\left(\Delta x^{\ell t+1}\right)$ and $\frac{\omega_{k}^{Q}-\delta_{k}}{\delta_{k}}=\mathcal{O}\left(\Delta x^{\ell t+1}\right)$. Finally, the sufficient conditions of Lemma 2 for optimal convergence order are satisfied if

$$
\ell(\theta(\tau)-\hat{m})+1 \geq G-g \quad \text { and } \quad \ell(\theta(\tau)-\hat{m})+1 \geq G-\gamma_{k}-r_{k}, k=1, \ldots, n
$$

or equivalently

$$
\ell(\theta(\tau)-\hat{m}) \geq \max \left\{G-g-1, \max _{k=1, \ldots, n}\left\{G-\gamma_{k}-r_{k}-1\right\}\right\} .
$$

Case $(B): \boldsymbol{n}_{\boldsymbol{c} p}<\boldsymbol{g}$ Proposition 2 applied to $P_{0}(x), \ldots, P_{m}(x)$ with $M=g$ ensures that $R_{k}=o\left(B_{g}\right)$ and thus, for $k=0, \ldots, m$,

$\alpha_{k}^{P}=d_{k}\left(1+\left(a_{k}\right)^{\ell}\right) \quad$ with $\quad a_{k}=\frac{\tau}{B_{g}+\epsilon} \frac{1}{1+\frac{R_{k}}{B_{g}+\epsilon}} \sim \frac{\tau}{B_{g}+\epsilon} \sim \frac{C_{\tau} \Delta x^{\theta(\tau)}}{C_{g} \Delta x^{2 n_{c p}+2}+C_{\epsilon} \Delta x^{\hat{m}}}$.

Similarly, for $k=1, \ldots, n$, we can use Proposition 1 to obtain

$$
\alpha_{k}^{Q}=\delta_{k}\left(1+\left(b_{k}\right)^{\ell}\right) \quad \text { with } \quad b_{k}=\frac{\tau}{I_{k}^{Q}+\epsilon} \sim \frac{C_{\tau} \Delta x^{\theta(\tau)}}{\widehat{C}_{k} \Delta x^{2 n_{c p}+2}+C_{\epsilon} \Delta x^{\hat{m}}} .
$$


We observe that if $2 n_{c p}+2>\hat{m}$, i.e. $\frac{\hat{m}-2}{2}<n_{c p}<g$, then $a_{k}, b_{k} \sim C \Delta x^{\theta(\tau)-\hat{m}} \rightarrow 0$ with $C=C_{\tau} / C_{\epsilon}$ independent on $k$. Consequently, we are in the same situation as Case (A) and we can apply Lemma 3 and Lemma 2 to prove the sufficient conditions for optimal convergence given in (12).

In particular, $\hat{m}=1$ is always included in the analysis above. Instead, we must distinguish some sub-cases when $0 \leq n_{c p} \leq \frac{\hat{m}-2}{2}, \hat{m}>1$.

Case (B1): $\boldsymbol{n}_{\boldsymbol{c} \boldsymbol{p}}=\frac{\hat{\boldsymbol{m}}-\mathbf{2}}{\mathbf{2}}, \hat{\boldsymbol{m}} \geq \mathbf{2}$ In this case, defining $t=\theta(\tau)-\hat{m}$, we have

$$
a_{k} \sim \frac{C_{\tau}}{C_{g}+C_{\epsilon}} \Delta x^{t} \quad \text { and } \quad b_{k} \sim \frac{C_{\tau}}{\widehat{C}_{k}+C_{\epsilon}} \Delta x^{t} .
$$

We cannot directly apply Lemma 3 , but, defining $C=\frac{C_{\tau}}{C_{g}+C_{\epsilon}}$ and $\widetilde{C}_{k}=\frac{C_{\tau}}{\widehat{C}_{k}+C_{\epsilon}}$, we compute

$$
\begin{aligned}
\sum_{k=0}^{m} \alpha_{k}^{P}+\sum_{k=1}^{n} \alpha_{k}^{Q} & =\sum_{k=0}^{m} d_{k}\left(1+C^{\ell} \Delta x^{\ell t}\right)+\sum_{k=1}^{n} \delta_{k}\left(1+\widetilde{C}_{k}^{\ell} \Delta x^{\ell t}\right) \\
& =1+\left(1-\sum_{k=1}^{n} \delta_{k}\right) C^{\ell} \Delta x^{\ell t}+\sum_{k=1}^{n} \widetilde{C}_{k}^{\ell} \Delta x^{\ell t+r_{k}} \\
& \sim 1+C^{\ell} \Delta x^{\ell t} .
\end{aligned}
$$

Then, using (5), for $k=0, \ldots, m$ we compute

$$
\omega_{k}^{P} \sim d_{k}\left(1+C^{\ell} \Delta x^{\ell t}\right)\left(1-C^{\ell} \Delta x^{\ell t}\right)=d_{k}\left(1+\mathcal{O}\left(\Delta x^{\ell t+1}\right)\right)
$$

and similarly for $k=1, \ldots, n$ we have

$$
\omega_{k}^{Q} \sim \delta_{k}\left(1+\widetilde{C}_{k}^{\ell} \Delta x^{\ell t}\right)\left(1-C \Delta x^{\ell t}\right)=\delta_{k}\left(1+\mathcal{O}\left(\Delta x^{\ell t}\right)\right) .
$$

Thus, sufficient conditions of Lemma 2 for optimal convergence are satisfied if

$$
\ell(\theta(\tau)-\hat{m})+1 \geq G-g \quad \text { and } \quad \ell(\theta(\tau)-\hat{m}) \geq G-\gamma_{k}-r_{k}, k=1, \ldots, n
$$

or equivalently

$$
\ell(\theta(\tau)-\hat{m}) \geq \max \left\{G-g-1, \max _{k=1, \ldots, n}\left\{G-\gamma_{k}-r_{k}\right\}\right\} .
$$

Case (B2): $\boldsymbol{n}_{\boldsymbol{c} p}<\frac{\hat{\boldsymbol{m}}-\mathbf{2}}{\mathbf{2}}, \hat{\boldsymbol{m}} \geq \mathbf{3}$ The analysis of the previous cases was independent on the choice of the degrees $\gamma_{k}, k=1, \ldots, n$. Now, instead, we need to consider explicitly the case when a constant polynomial is present, say $\gamma_{1}, \ldots, \gamma_{n-1}>0$, while $\gamma_{n}=0$.

In this case, we have that

$$
a_{k} \sim \frac{C_{\tau}}{C_{g}+C_{\epsilon}} \Delta x^{t}, \quad b_{k} \sim \frac{C_{\tau}}{\widehat{C}_{k}+C_{\epsilon}} \Delta x^{t} \quad \text { and } \quad b_{n} \sim \frac{C_{\tau}}{C_{\epsilon}} \Delta x^{\widehat{t}}
$$

where we have defined $t=\theta(\tau)-2 n_{c p}-2$ and $\widehat{t}=\theta(\tau)-\hat{m}$. Notice that $t>\widehat{t}$. 
Defining $C=\frac{C_{\tau}}{C_{g}+C_{\epsilon}}, \widetilde{C}_{k}=\frac{C_{\tau}}{\widehat{C}_{k}+C_{\epsilon}}$ and $\widetilde{C}_{n}=\frac{C_{\tau}}{C_{\epsilon}}$, we have that

$$
\begin{aligned}
\sum_{k=0}^{m} \alpha_{k}^{P}+\sum_{k=1}^{n} \alpha_{k}^{Q} & =\sum_{k=0}^{m} d_{k}\left(1+C^{\ell} \Delta x^{\ell t}\right)+\sum_{k=1}^{n-1} \delta_{k}\left(1+\widetilde{C}_{k}^{\ell} \Delta x^{\ell t}\right)+\delta_{n}\left(1+\widetilde{C}_{n}^{\ell} \Delta x^{\ell \widehat{t}}\right) \\
& =1+\left(1-\sum_{k=1}^{n} \delta_{k}\right) C^{\ell} \Delta x^{\ell t}+\sum_{k=1}^{n-1} \tilde{C}_{k}^{\ell} \Delta x^{\ell t+r_{k}}+\left(1+\tilde{C}_{n}^{\ell} \Delta x^{\ell \widehat{t}+r_{n}}\right) \\
& \sim 1+C^{\ell} \Delta x^{\ell t}
\end{aligned}
$$

only if $r_{n}>\ell(t-\widehat{t})=\ell\left(\hat{m}-2 n_{c p}-2\right)$.

Then, using (5), for $k=0, \ldots, m$ we compute

$$
\omega_{k}^{P} \sim d_{k}\left(1+C^{\ell} \Delta x^{\ell t}\right)\left(1-C^{\ell} \Delta x^{\ell t}\right)=d_{k}\left(1+\mathcal{O}\left(\Delta x^{\ell t+1}\right)\right)
$$

and similarly for $k=1, \ldots, n$ we have

$$
\omega_{k}^{Q} \sim \delta_{k}\left(1+\widetilde{C}_{k}^{\ell} \Delta x^{\ell t}\right)\left(1-C \Delta x^{\ell t}\right)=\delta_{k}\left(1+\mathcal{O}\left(\Delta x^{\ell t}\right)\right)
$$

for $k=1, \ldots, n-1$ and

$$
\omega_{n}^{Q} \sim \delta_{k}\left(1+\widetilde{C}_{n}^{\ell} \Delta x^{\ell \widehat{t}}\right)\left(1-C \Delta x^{\ell t}\right)=\delta_{n}\left(1+\mathcal{O}\left(\Delta x^{\ell \widehat{t}}\right)\right) .
$$

Thus, sufficient conditions of Lemma 2 for optimal convergence are satisfied if $\ell\left(\theta(\tau)-2 n_{c p}-2\right)+1 \geq G-g, \quad \ell\left(\theta(\tau)-2 n_{c p}-2\right) \geq G-\gamma_{k}-r_{k}, k=1, \ldots, n-1$ and

$$
\ell(\theta(\tau)-\hat{m}) \geq G-r_{n}
$$

In the general case these conditions become

$$
\begin{array}{r}
\ell\left(\theta(\tau)-2 n_{c p}-2\right) \geq \max \left\{G-g-1, \max _{\left\{k: \gamma_{k}>0\right\}}\left\{G-\gamma_{k}-r_{k}\right\}\right\} \\
\ell(\theta(\tau)-\hat{m}) \geq G-r_{k}, \quad\left\{k: \gamma_{k}=0\right\} .
\end{array}
$$

Summarizing, relations (11), (12), (13) and (14) define the complete set of sufficient conditions for optimal convergence. In particular, once (11) is applied in order to find an

Table 1 Summary of the sufficient conditions, and related cases, for optimal convergence depending on the values of $n_{c p} \leq 4$ and $\hat{m} \leq 4$

\begin{tabular}{llllll}
\hline \multicolumn{1}{c}{$n_{c p}$} & & & & \\
\hline$\hat{m}$ & 0 & 1 & 2 & 3 & 4 \\
1 & & & & & \\
& Eq. (12) & Eq. (12) & Eq. (12) & Eq. (12) & Eq. (12) \\
2 & (A)-(B) & (A)-(B) & (A)-(B) & (A)-(B) & (A)-(B) \\
& Eq. (13) & Eq. (12) & Eq. (12) & Eq. (12) & Eq. (12) \\
3 & $($ B1) & (A)-(B) & (A)-(B) & (A)-(B) & (A)-(B) \\
& Eq. (14) & Eq. (12) & Eq. (12) & Eq. (12) & Eq. (12) \\
4 & (B2) & (A)-(B) & (A)-(B) & (A)-(B) & (A)-(B) \\
& Eq. (14) & Eq. (13) & Eq. (12) & Eq. (12) & Eq. (12) \\
& (B2) & (B1) & (A)-(B) & (A)-(B) & (A)-(B) \\
\hline
\end{tabular}


upper bound for $\hat{m}$, conditions on $\ell$ and $r_{k}$ can be found. We summarize them in Table 1 and for some prototype reconstructions in the following section.

\subsection{A 7-th Order CWENOZ-AO Reconstruction}

The definition of the CWENOZ-AO reconstruction, see Definition 5, allows to define a very wide set of reconstructions characterized by a very high order gap between the optimal polynomial and lowest degree polynomials. Moreover, we stress the fact that CWENOZ-AO does not require a base level reconstruction, therefore sharing the feature of the WENO-AO reconstruction by [2].

Here, we propose one adaptive order reconstruction of order 7 which we name CWZ753 and it will be later numerically compared with the WENO-AO reconstructions of order 7 introduced in [2,5]. The CWZ753 is characterized by a stencil $\mathcal{S}_{\text {opt }}$ of seven cells centered on the computational cell $\Omega_{0}$ which is the same stencil on which the WENO-AO reconstructions of [2] and [5] are built. The optimal polynomial $P_{\text {opt }} \in \mathbb{P}^{6}$ interpolates the data in $\mathcal{S}_{\text {opt }}$ and the polynomial $P_{1} \in \mathbb{P}^{4}$ interpolates the data of the five central cells of $\mathcal{S}_{\text {opt }}$. The lower degree polynomials $Q_{1}, Q_{2}, Q_{3} \in \mathbb{P}^{2}$ are the parabolas interpolating the data of the three substencils $\left\{\Omega_{k-3}, \Omega_{k-2}, \Omega_{k-1}\right\}$ for $k=1,2,3$. Consequently, in the notation of Definition 5, we are in the case of $G=6, g=4$ and $\gamma_{k}=2$, for $k=1,2,3$, and the reconstruction polynomial is defined by

$$
P_{\text {rec }}=\operatorname{CWENOZ-AO}\left(P_{\text {opt }} ; P_{1} ; Q_{1}, Q_{2}, Q_{3}\right) \in \mathbb{P}^{6} .
$$

We employ as global smoothness indicator $\tau=\left|I_{0}-I\left[P_{1}\right]\right|$. Let $u=u(x)$ be a function continuously differentiable with $x \in \mathbb{R}$, and let $\Omega_{0}=\left[-\frac{\Delta x}{2}, \frac{\Delta x}{2}\right]$, then the Taylor expansion of the global smoothness indicator $\tau$ is

$$
\begin{aligned}
\tau= & \frac{1}{15} u^{\prime}(0) u^{(5)}(0) \Delta x^{6} \\
& +\left[\frac{1}{40} u^{\prime}(0) u^{(7)}(0)+\frac{31}{1260} u^{\prime \prime}(0) u^{(6)}(0)-\frac{16403}{30240} u^{\prime \prime \prime}(0) u^{(5)}(0)\right] \Delta x^{8} \\
& +\mathcal{O}\left(\Delta x^{10}\right) .
\end{aligned}
$$

We use it to compute the parameters $\hat{m}, \ell$ and $r_{k}, k=1,2,3$, satisfying the sufficient conditions for optimal convergence. All the values, for each $n_{c p}$ and $\hat{m}$, are summarized in Table 2 by using the smaller value allowed for $g$, i.e. $g=\frac{G}{2}=3$. Condition (11) provides the restriction $\hat{m} \leq 7$.

Remark 2 Both in [5] and [2], a WENO-AO reconstruction of order 9 is considered, where the base level is again represented by the three parabolas as in the $\operatorname{WENO}-\mathrm{AO}(7,5,3)$ reconstruction. The ninth-order scheme computes a reconstruction polynomial as

$$
P_{\text {rec }}=\operatorname{CWENOZ}\left(\operatorname{CWENOZ}\left(P^{(9)} ; P_{L}^{(3)}, P_{C}^{(3)}, P_{R}^{(3)}\right) ; \operatorname{WENO}-A O(7,5,3)\right) .
$$

The corresponding reconstruction based on the CWENOZ-AO definition would compute the reconstruction polynomial as

$$
P_{\text {rec }}=\operatorname{CWENOZ-AO}\left(P^{(9)} ; P^{(7)}, P^{(5)} ; P_{L}^{(3)}, P_{C}^{(3)}, P_{R}^{(3)}\right) .
$$

Comparing (16) and (17), we observe that CWENOZ-AO always computes one set of nonlinear coefficients. The WENO-AO scheme of [5] needs five sets of nonlinear weights, while the WENO-AO scheme of [2] requires three sets of nonlinear coefficients. It is then clear that, 
Table 2 Sufficient conditions for optimal convergence for the reconstruction CWZ753 defined in Sect. 3.2 and by Eq. (15)

\begin{tabular}{|c|c|c|c|c|c|}
\hline & \multicolumn{4}{|l|}{$n_{c p}$} & \multirow[t]{2}{*}{ Summary } \\
\hline & $\begin{array}{l}0 \\
\theta(\tau)=6\end{array}$ & $\begin{array}{l}1 \\
\theta(\tau)=8\end{array}$ & $\begin{array}{l}2 \\
\theta(\tau)=8\end{array}$ & $\begin{array}{l}\geq 3 \\
\theta(\tau)=10\end{array}$ & \\
\hline \multicolumn{6}{|c|}{$\hat{m}$} \\
\hline 1 & $\ell \geq 1, r_{k} \geq 1$ & $\ell \geq 1, r_{k} \geq 1$ & $\ell \geq 1, r_{k} \geq 1$ & $\ell \geq 1, r_{k} \geq 1$ & $\ell \geq 1, r_{k} \geq 1$ \\
\hline 2 & $\ell \geq 1, r_{k} \geq 1$ & $\ell \geq 1, r_{k} \geq 1$ & $\ell \geq 1, r_{k} \geq 1$ & $\ell \geq 1, r_{k} \geq 1$ & $\ell \geq 1, r_{k} \geq 1$ \\
\hline 3 & $\ell \geq 1, r_{k} \geq 1$ & $\ell \geq 1, r_{k} \geq 1$ & $\ell \geq 1, r_{k} \geq 1$ & $\ell \geq 1, r_{k} \geq 1$ & $\ell \geq 1, r_{k} \geq 1$ \\
\hline 4 & $\ell \geq 1, r_{k} \geq 1$ & $\ell \geq 1, r_{k} \geq 1$ & $\ell \geq 1, r_{k} \geq 1$ & $\ell \geq 1, r_{k} \geq 1$ & $\ell \geq 1, r_{k} \geq 1$ \\
\hline 5 & $\ell \geq 1, r_{k} \geq 1$ & $\ell \geq 1, r_{k} \geq 1$ & $\ell \geq 1, r_{k} \geq 1$ & $\ell \geq 1, r_{k} \geq 1$ & $\ell \geq 1, r_{k} \geq 1$ \\
\hline 6 & $\ell \geq 1, r_{k} \geq 1$ & $\ell \geq 1, r_{k} \geq 1$ & $\begin{array}{l}\ell \geq 2, r_{k} \geq 1 \\
\ell \geq 1, r_{k} \geq 2\end{array}$ & $\ell \geq 1, r_{k} \geq 1$ & $\begin{array}{l}\ell \geq 2, r_{k} \geq 1 \\
\ell \geq 1, r_{k} \geq 2\end{array}$ \\
\hline 7 & $\ell \geq 1, r_{k} \geq 1$ & $\ell \geq 1, r_{k} \geq 1$ & $\begin{array}{l}\ell \geq 3, r_{k} \geq 1 \\
\ell \geq 2, r_{k} \geq 2\end{array}$ & $\ell \geq 1, r_{k} \geq 1$ & $\begin{array}{l}\ell \geq 3, r_{k} \geq 1 \\
\ell \geq 2, r_{k} \geq 2\end{array}$ \\
\hline
\end{tabular}

increasing the order, the gap between the computational cost required by the CWENOZ-AO and the WENO-AO reconstructions becomes wider.

\section{Numerical Experiments}

Here we numerically test the performance of the adaptive order CWENOZ-AO scheme of order 7 proposed in Sect. 3.2. This reconstruction will be referred to as CWZ753 in this section. We compare it with the two WENO-AO reconstructions of order 7 reviewed in Sect. 2.1.2. For convenience, they will be referred to as WAO-AHZ for the reconstruction of [2] and WAO-BGS for the reconstruction of [5]. The study is performed in terms of accuracy and computational cost.

First, in Sect. 4.1 we test the optimal order of convergence of the novel schemes for several choices of the parameters, with the aim of providing a numerical evidence supporting the convergence results of Sect. 3.1. In Sect. 4.2, the non-oscillatory properties will be analyzed on the linear advection of a non-smooth datum. Next, in Sect. 4.3 we consider one-dimensional test problems based on the system of Euler equations for gas dynamics. Finally, in Sect. 4.4 we show the performance of the schemes for the solution of a system of balance laws. In Sect. 4.5 all these experiments are supported by comparisons on the computational cost required by the different schemes.

For the numerical solution we set up a finite volume scheme of order 7 on a uniform grid, based on the method of lines and the seventh order Runge-Kutta scheme with nine stages [8, p. 196]. At each Runge-Kutta stage, the cell averages are used to compute the reconstructions and the boundary extrapolated data are fed into the Local Lax-Friedrichs numerical flux. The source term and the initial data are computed with the four point Gaussian quadrature of order seven. All the simulations are run with a CFL of 0.9 , unless otherwise stated.

The CWENOZ-AO reconstruction employs $\delta_{k}=\min \left(\Delta x^{r_{k}}, 0.01\right)$ for $k=1, \ldots, n$, $d_{1}=0.15$ and a central optimal weight $d_{0}=0.85-\sum_{k=1}^{n} \delta_{k}$. The values of $\hat{m}, \ell$ and $r_{k}, k=1, \ldots, n$, are specified in each test. Instead, for the numerical solution of systems 
of conservation and balance laws we do not perform any tuning on the parameters and we consider $\hat{m}=4, \ell=2$ and $r_{k}=1, \forall k$. The WENO-AO reconstructions are implemented as described in [2], and used with the same set of parameters $\hat{m}$ and $\ell$ specified therein.

All computations have been performed with the claw1dArena open-source software [38].

\subsection{Accuracy of the Reconstructions}

For the accuracy tests we consider the following functions and critical points:

\begin{tabular}{lll}
\hline$n_{c p}$ & Function & $x_{\text {crit }}$ \\
\hline 0 & $u_{0}(x)=e^{-x^{2}}$ & 0.2 \\
1 & $u_{1}(x)=\sin (\pi x-\sin (\pi x) / \pi)$ & 0.596683186911209 \\
2 & $u_{2}(x)=1.0+\sin ^{3}(\pi x)$ & 0.0 \\
\hline
\end{tabular}

Obviously, for $n_{c p}=0, x_{\text {crit }}=0.2$ is an evaluation point rather than a critical point. We compute the reconstruction polynomial for the cell containing the critical point, and the cell averages in the stencil are initialized with the Gauss-Legendre quadrature rule with 4 nodes. For these tests, quadruple precision has been used. We aim to study numerically the conditions for the optimal convergence discussed in Sect. 3.1.1.

In Table $3 \mathrm{a}, \mathrm{b}$ we show reconstruction errors and convergence rates for the parameter values $\hat{m}=4, \ell=2$ and $r_{k}=1, \forall k$, and which are used in the following numerical experiments, on critical points of order $n_{c p}=0$ and $n_{c p}=1$, respectively. The optimal order of reconstruction is reached already on coarse grids, and we observe a similar behavior for any $n_{c p} \geq 2$.

In Table $3 \mathrm{c}$, $\mathrm{d}$ we focus on $n_{\mathrm{cp}}=2$, since this value induces a restriction on the parameters $\ell$ and $r_{k}$ when $\hat{m}=6,7$, see Table 2 . In particular, in Table $3 \mathrm{c}$ we consider a set of parameters, $\hat{m}=6, \ell=1$ and $r_{k}=1$, which does not satisfy the sufficient conditions for optimal convergence. We observe that the order of convergence degrades to order 6 . Conversely, in Table $3 \mathrm{~d}$ the set of parameters, $\hat{m}=6, \ell=1$ and $r_{k}=2$, satisfy the sufficient conditions for optimal convergence and the optimal expected order is reached already on coarse grids.

This analysis shows that, although the conditions derived in Sect. 3.1.1 are only sufficient, they are rather strict since optimal order is degraded when these conditions are not fulfilled.

\subsection{Linear Transport Problem: Jiang-Shu Test}

We solve the linear scalar conservation law

$$
u_{t}+u_{x}=0
$$

on the periodic domain $x \in[-1,1]$ and up to final time $T=8$. As initial condition we consider the non-smooth profile 
Table 3 Reconstruction errors and order of convergence for CWZ753

\begin{tabular}{llllll}
\hline$\Delta x$ & Error & Rate & $\Delta x$ & Error & Rate \\
\hline (a) $\hat{m}=4, \ell=2, r=1$ on $n_{c p}=0$. & & (b) $\hat{m}=4, \ell=2, r=1$ on $n_{c p}=1$. & \\
0.1 & $1.04 \times 10^{-7}$ & - & 0.1 & $3.15 \times 10^{-5}$ & - \\
$5.00 \times 10^{-2}$ & $8.30 \times 10^{-10}$ & 6.97 & $5.00 \times 10^{-2}$ & $2.67 \times 10^{-7}$ & 6.88 \\
$2.50 \times 10^{-2}$ & $6.50 \times 10^{-12}$ & 7.00 & $2.50 \times 10^{-2}$ & $2.11 \times 10^{-9}$ & 6.98 \\
$1.25 \times 10^{-2}$ & $5.07 \times 10^{-14}$ & 7.00 & $1.25 \times 10^{-2}$ & $1.65 \times 10^{-11}$ & 7.00 \\
$6.25 \times 10^{-3}$ & $3.96 \times 10^{-16}$ & 7.00 & $6.25 \times 10^{-3}$ & $1.29 \times 10^{-13}$ & 7.00 \\
$3.13 \times 10^{-3}$ & $3.09 \times 10^{-18}$ & 7.00 & $3.13 \times 10^{-3}$ & $1.01 \times 10^{-15}$ & 7.00 \\
$1.56 \times 10^{-3}$ & $2.42 \times 10^{-20}$ & 7.00 & $1.56 \times 10^{-3}$ & $7.86 \times 10^{-18}$ & 7.00 \\
$7.81 \times 10^{-4}$ & $1.89 \times 10^{-22}$ & 7.00 & $7.81 \times 10^{-4}$ & $6.14 \times 10^{-20}$ & 7.00 \\
$(\mathrm{c}) \hat{m}=6, \ell=1, r=1$ on $n_{c p}=2$. & & $(\mathrm{d}) \hat{m}=6, \ell=1, r=2$ on $n_{c p}=2$. & \\
0.1 & $8.14 \times 10^{-3}$ & - & 0.1 & $8.14 \times 10^{-3}$ & - \\
$5.00 \times 10^{-2}$ & $4.88 \times 10^{-4}$ & 4.06 & $5.00 \times 10^{-2}$ & $1.54 \times 10^{-4}$ & 5.72 \\
$2.50 \times 10^{-2}$ & $1.76 \times 10^{-5}$ & 4.79 & $2.50 \times 10^{-2}$ & $1.21 \times 10^{-6}$ & 6.99 \\
$1.25 \times 10^{-2}$ & $5.59 \times 10^{-7}$ & 4.98 & $1.25 \times 10^{-2}$ & $9.17 \times 10^{-9}$ & 7.05 \\
$6.25 \times 10^{-3}$ & $1.10 \times 10^{-8}$ & 5.67 & $6.25 \times 10^{-3}$ & $7.09 \times 10^{-11}$ & 7.02 \\
$3.13 \times 10^{-3}$ & $1.71 \times 10^{-10}$ & 6.00 & $3.13 \times 10^{-3}$ & $5.52 \times 10^{-13}$ & 7.00 \\
$1.56 \times 10^{-3}$ & $2.67 \times 10^{-12}$ & 6.00 & $1.56 \times 10^{-3}$ & $4.31 \times 10^{-15}$ & 7.00 \\
$7.81 \times 10^{-4}$ & $4.18 \times 10^{-14}$ & 6.00 & $7.81 \times 10^{-4}$ & $3.37 \times 10^{-17}$ & 7.00 \\
\hline
\end{tabular}

$$
u_{0}(x)= \begin{cases}\frac{1}{6}(G(x, \beta, z-\delta)+G(x, \beta, z+\delta)+4 G(x, \beta, z)), & -0.8 \leq x \leq-0.6, \\ 1, & -0.4 \leq x \leq-0.2, \\ 1-|10(x-0.1)|, & 0 \leq x \leq 0.2, \\ \frac{1}{6}(F(x, \alpha, a-\delta)+F(x, \alpha, a+\delta)+4 F(x, \alpha, a)), & 0.4 \leq x \leq 0.6 \\ 0, & \text { otherwise }\end{cases}
$$

where

$$
G(x, \beta, z)=\exp \left(-\beta(x-z)^{2}\right), \quad F(x, \alpha, a)=\sqrt{\max \left\{1-\alpha^{2}(x-a)^{2}, 0\right\}}
$$

and the constants are taken as $a=0.5, z=-0.7, \delta=0.005, \alpha=10$ and $\beta=\log 2 / 36 \delta^{2}$. This problem, designed by Jiang and Shu in [28], is used in order to investigate the properties of a scheme to transport different shapes with minimal dissipation and dispersion effects. The initial condition (18) is a combination of smooth and non-smooth shapes: precisely, from the left to the right side of the domain, we have a Gaussian, a square wave, a sharp triangle wave and a half ellipse.

Figure 1 shows the numerical solutions of the Jiang and Shu test problem computed with the CWENOZ-AO and the WENO-AO schemes of order 7 on 400 cells. In particular, zoom on the top part of the Gaussian wave and of the square wave and zoom on the bottom part of the square wave are considered in order to give information on the behavior of the schemes on smooth and non-smooth zones of the solution. We observe that all the schemes 
Jiang-Shu test

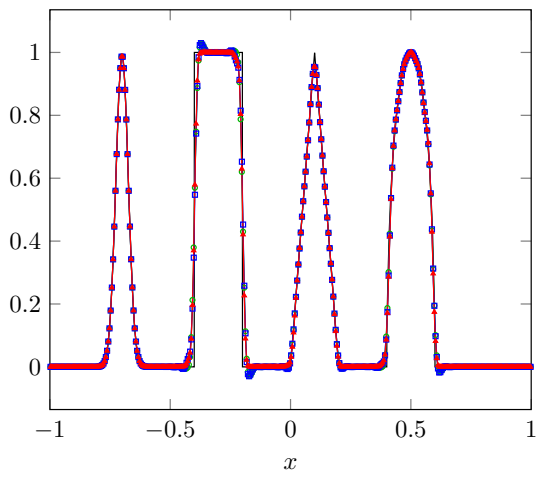

zoom top square wave

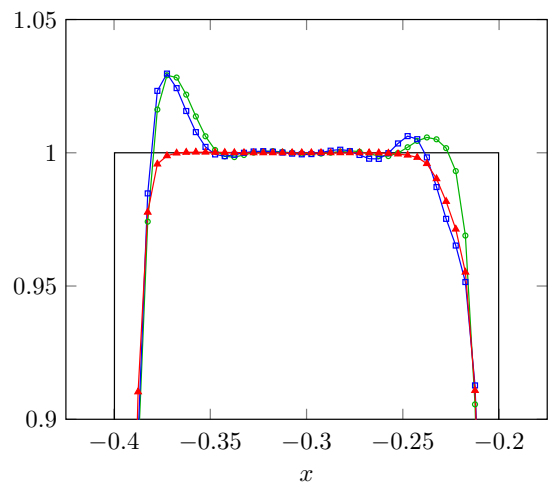

zoom top Gaussian

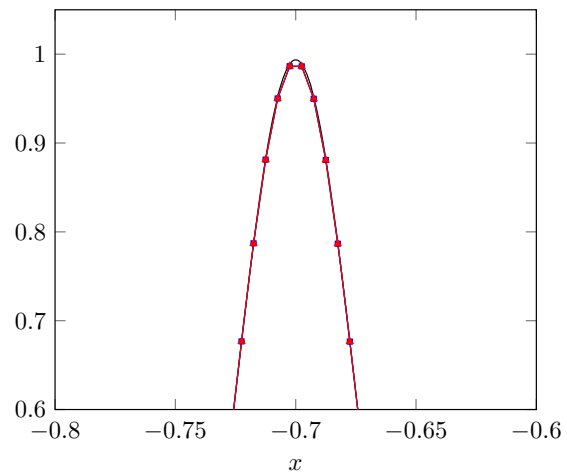

zoom bottom square wave

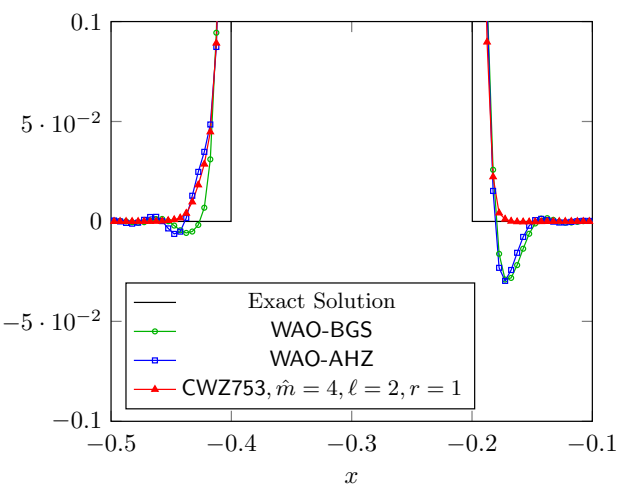

Fig. 1 Numerical solution of the Jiang-Shu test problem (18) with schemes of order 7 and 400 cells, with zoom on the top part of the Gaussian wave, the top and the bottom part of the square wave

perform similarly and without significant difference on smooth zones. However, compared to WENO-AO, the novel adaptive order reconstruction introduced in this work presents less oscillations close to discontinuities. This is made possible by the very small value of $\epsilon$ (large $\hat{m}$ ) which allows to damp the spurious oscillations. Taking $\hat{m}=3$ we observe small overshoots and undershoots, but their amplitude is still less than the amplitude of the oscillations produced by the WENO-AO schemes. Choices of $\hat{m}<3$ lead to more oscillating solutions.

\subsection{Euler Equations}

We consider the one-dimensional system of Euler equations for gas dynamics

$$
\frac{\partial}{\partial t}\left(\begin{array}{c}
\rho \\
\rho u \\
E
\end{array}\right)+\frac{\partial}{\partial x}\left(\begin{array}{c}
\rho u \\
\rho u^{2}+p \\
u(E+p)
\end{array}\right)=0,
$$

where $\rho, u, p$ and $E$ are the density, velocity, pressure and energy per unit volume of an ideal gas, whose equation of state is $E=\frac{p}{\gamma-1}+\frac{1}{2} \rho u^{2}$, where $\gamma=1.4$. 

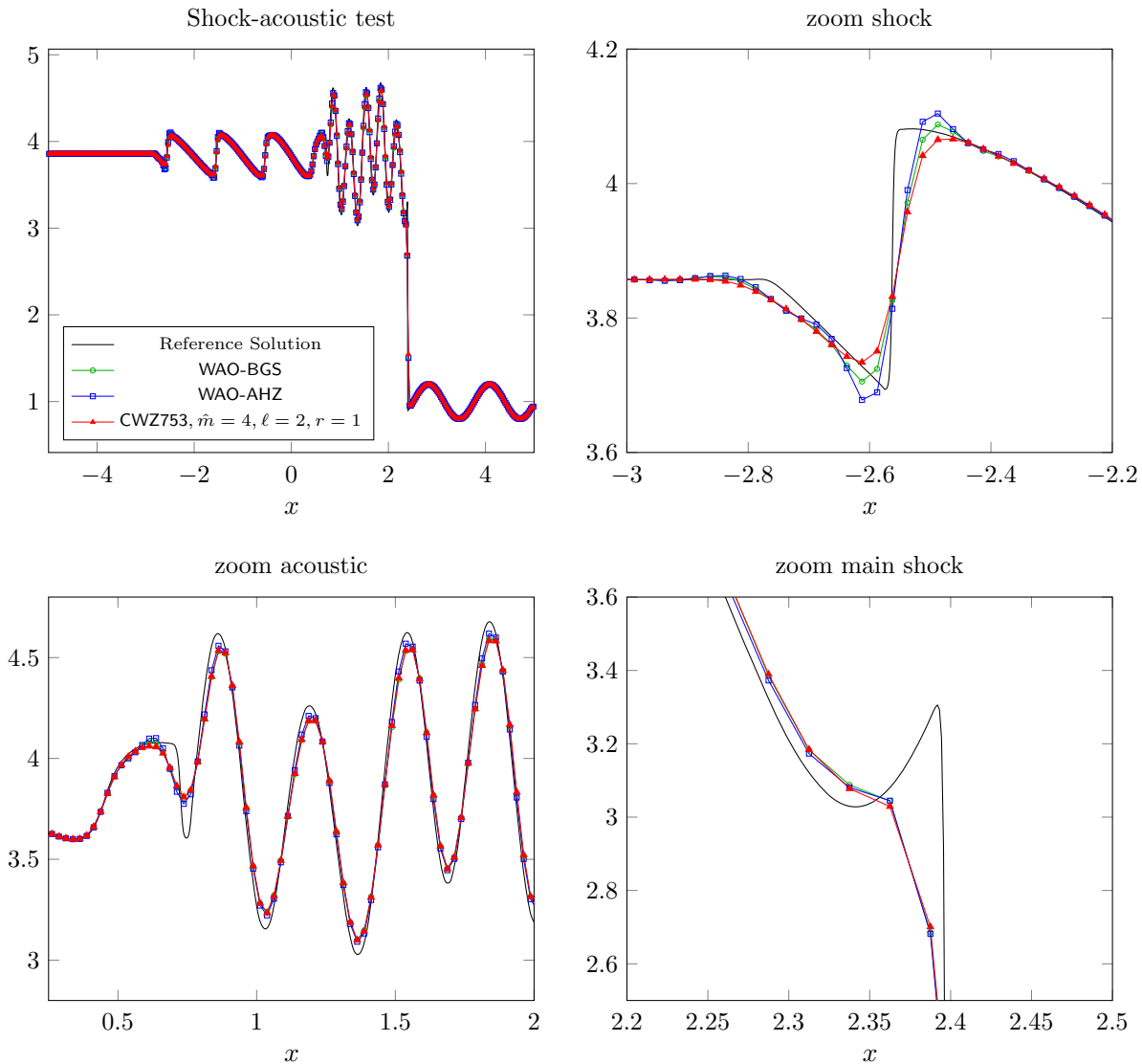

Fig. 2 Numerical solution of the shock-acoustic wave interaction problem with schemes of order 7 on 400 cells and zoom on three regions of the density profile. Reconstructions are performed along characteristic variables

Shock-acoustic interaction problem. This consists in computing the interaction of a strong shock with an acoustic wave on the domain $x \in[-5,5]$ with free-flow boundary conditions. The problem was introduced by Shu and Osher in [42] and is characterized by a Mach 3 shock wave interacting with a standing sinusoidal density wave. The solution, behind the main strong shock, develops a combination of smooth waves and small discontinuities. The initial condition is

$$
(\rho, u, p)= \begin{cases}(3.857143,2.629369,10.333333), & x<-4 \\ (1+0.2 \sin (5 x), 0,1), & x \geq-4\end{cases}
$$

and we run the problem up to the final time $T=1.8$.

Figure 2 shows the numerical results computed with the CWENOZ-AO and the two WENO-AO schemes of order 7 on 400 cells using a CFL of 0.75 . We consider the zoom-in of the solution in three regions of the computational domain: the first shocklet, the turbulence zone characterized by the smooth high-frequency solution behind the main shock and the main shock itself. The reference solution (black line) was generated using 8000 cells and the third 
order CWENO scheme. All the reconstructions are computed along characteristic variables. In any case, it should be noted that a local application of the local characteristic projection in few cells around the discontinuities should be enough [34,35]. We observe that all the schemes provide very similar accuracy on the smooth region, where the WAO-AHZ scheme has a slight better resolution close to the extrema. We observe instead different behaviors in the approximation of the first shock in the shocklets region. The CWZ753 reconstruction is more diffusive, avoiding the small oscillations produced by the WENO-AO schemes. Note that, instead, no extra oscillations at the main shock, which is very underresolved, are observed using any of the schemes.

Lax test. We solve the Riemann problem by Lax which is characterized by following initial states:

$$
(\rho, u, p)= \begin{cases}(0.445,0.6989,3.5277), & x<0.5 \\ (0.5,0,0.571), & x \geq 0.5\end{cases}
$$

up to final time $T=0.15$. The solution develops a rarefaction wave traveling left, a contact discontinuity and a shock, both with positive speeds. This test is challenging when solved by high-order schemes which might produce spurious oscillations on the density peak, between the contact discontinuity and the shock. The oscillations are originated by the interaction between waves in the first stages of the solution, when the discontinuities are so close that the algorithm cannot find a smooth stencil. They can be partly cured computing the reconstruction along characteristic fields, where the waves are approximately decoupled [36]. Adaptive order reconstructions being able to include low order base levels can also help to reduce oscillations, since smooth stencils can be used.

In Fig. 3 we show the numerical solution provided by the CWENOZ-AO and the WENO-AO schemes of order 7 on a grid of $N=400$ cells. We consider the zoom-in on the top of the rarefaction wave, on the lowest density point corresponding to the bottom of the contact discontinuity and, finally, on the density peak. The solution of the Riemann problem at final time (black line) is computed exactly [43]. All the schemes use reconstructions along characteristic variables. We observe that the WENO-AO scheme given in [5] produces small oscillations in the three regions, while the WENO-AO scheme given in [2] produces a small overshoot only around the top of the rarefaction wave. Instead, the CWENOZ-AO reconstructions does not develop spurious oscillations, even if it is less accurate than the WENO-AO scheme given in [2] on the bottom part of the contact discontinuity.

\subsection{Balance Laws: Euler Equations in Spherical Symmetry}

In the case of radial symmetry, the multi-dimensional gas dynamics equations can be written as a one-dimensional system, with a source term, which takes into account the geometrical effect, $[43, \S 1.6 .3]$. In radial symmetry all the variable are functions of the time $t$ and the radial distance from the origin $\sigma$. Radially symmetric solutions of the Euler equations in $\mathbb{R}^{d}$ may be computed by solving

$$
\frac{\partial}{\partial t}\left(\begin{array}{c}
\rho \\
\rho u \\
E
\end{array}\right)+\frac{\partial}{\partial \sigma}\left(\begin{array}{c}
\rho u \\
\rho u^{2}+p \\
u(E+p)
\end{array}\right)=-\frac{d-1}{\sigma}\left(\begin{array}{c}
\rho u \\
\rho u^{2} \\
u p
\end{array}\right)
$$


Lax test

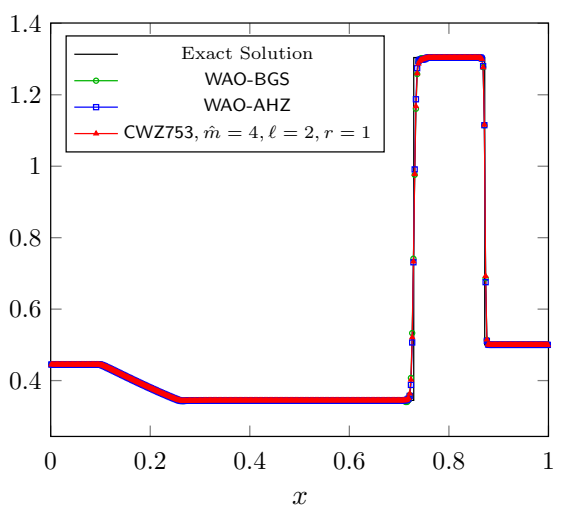

zoom lowest density

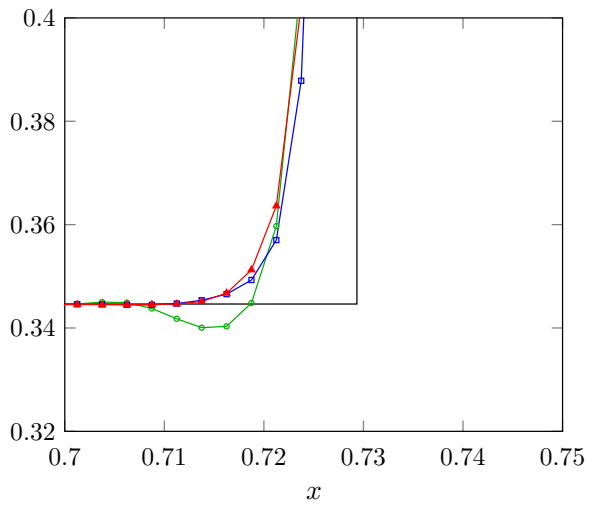

zoom rarefaction

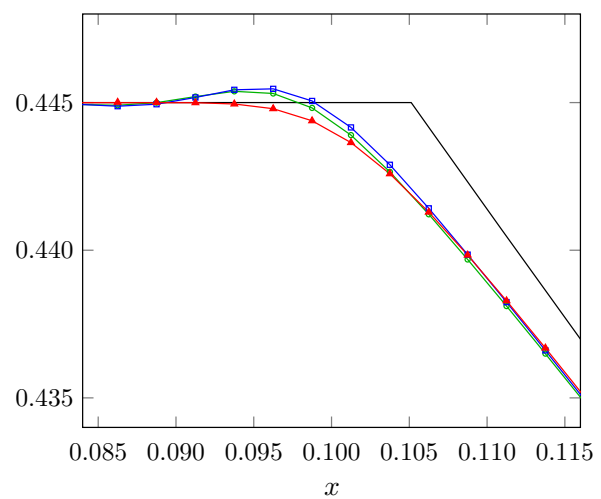

zoom density peak

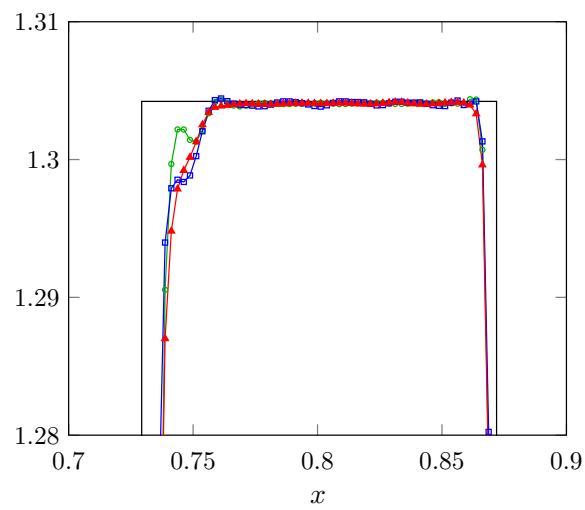

Fig. 3 Numerical solution of the Lax test problem with schemes of order 7 on 400 cells and zoom on three regions of the density profile. Reconstructions are performed along characteristic variables

where $\rho, u, p$ and $E$ are density, radial velocity, pressure and energy per unit volume of an ideal gas, whose equation of state is still specified by $E=\frac{p}{\gamma-1}+\frac{1}{2} \rho u^{2}$, with $\gamma=1.4$. When $d=2$ we have cylindrical symmetry, an approximation to two-dimensional flow. When $d=3$ we have spherical symmetry, an approximation to three-dimensional flow.

We solve the so-called "explosion problem" in three space dimensions, which has a shock tube like initial data. In our case, we take Sod's test data, namely

$$
(\rho, u, p)= \begin{cases}(1,0,1), & \sigma<0.5 \\ (0.125,0,0.1), & \sigma>0.5\end{cases}
$$

The final time of the simulation is $T=0.5$. We compute the solution for $\sigma \in[0,1]$ with wall boundary conditions. The Gaussian quadrature formulas of order 7 is employed to compute the cell average of the source term, which also avoids quadrature nodes at $\sigma=0$.

The density profile for $d=3$ at final time obtained with $N=400$ cells using the CWENOZ-AO and the two WENO-AO schemes is shown in Fig. 4, restricted to the domain $\sigma \in[0,1]$. The zoom in the density profile are centered on the bottom part of the discontinuity before the density peak, on the density peak and on the wave between the density peak and the 
Spherical Sod test

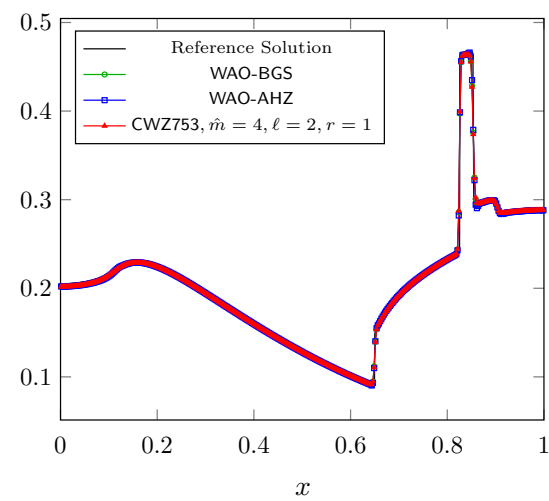

zoom density peak

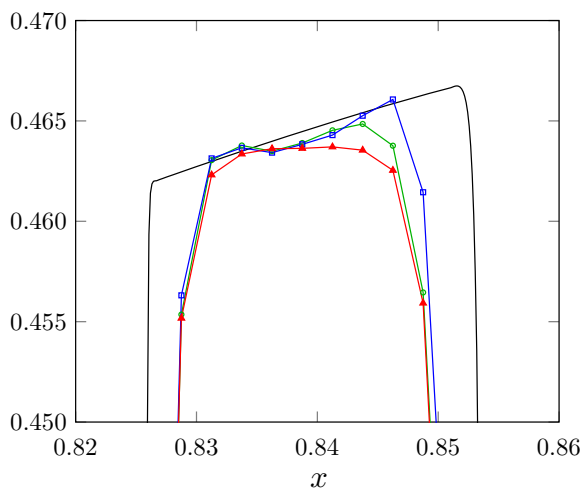

zoom 1

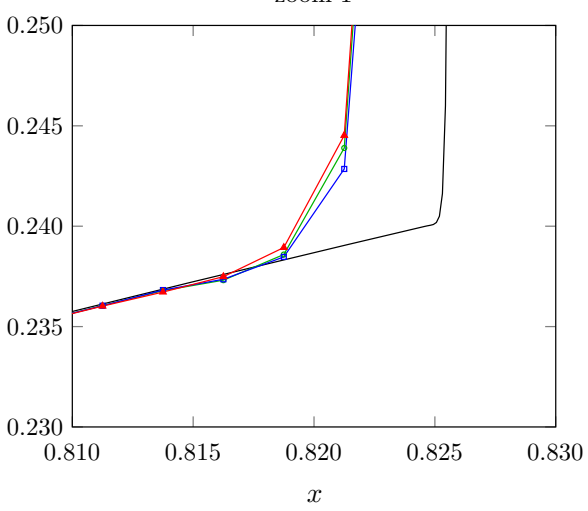

zoom 2

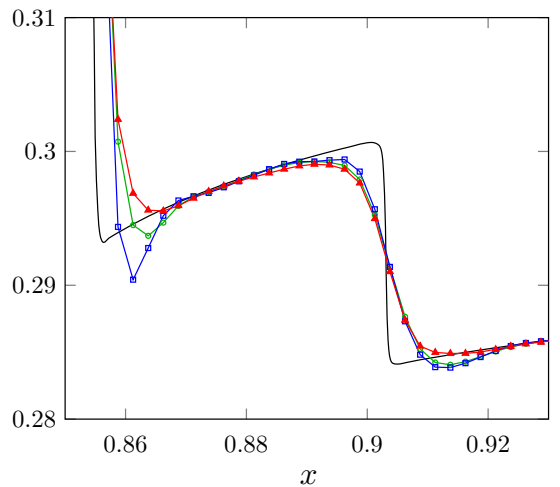

Fig. 4 Numerical solution of the radially spherical Sod's explosion problem with schemes of order 7 with 400 cells and zoom on three regions of the density profile. Reconstructions are performed along characteristic variables

right boundary. All the reconstructions are computed along characteristic variables in order to avoid spurious oscillations. We observe that the CWENOZ-AO scheme is more diffusive in the approximation of the discontinuities around the density peak. This is due to the choice of $\hat{m}=4$ and we recall that we do not perform tuning on the parameters. Actually, in this region of the density profile we observe a better accuracy of the CWENOZ-AO reconstruction when using $\hat{m}=2$. However, with $\hat{m}=4$ we can avoid the undershoots exhibited by the WENO-AO reconstructions, see the right most panel of Fig. 4.

\subsection{Computational Efficiency}

All the numerical experiments have shown that the adaptive order CWENOZ-AO scheme of order 7 performs similarly to the two WENO-AO schemes of order 7 in terms of accuracy and that their parameters may often be tuned to obtain less oscillatory reconstructions. This is not surprising since these schemes share the same idea of nonlinearly blending reconstruction polynomials which cover very high order gap. However, as already discussed in Sect. 3, the CWENOZ-AO scheme is defined in such a way that the definition of intermediate 
Table 4 Comparison of the computational times

\begin{tabular}{|c|c|c|c|c|c|c|}
\hline \multirow[b]{2}{*}{ Cells } & \multicolumn{3}{|c|}{ Core i7-6600U @ 2.60GHz } & \multicolumn{3}{|c|}{ Corei3-2100T @2.50GHz } \\
\hline & CWZ753(s) & WAO-BGS(\%) & WAO-AHZ(\%) & CWZ753(s) & WAO-BGS(\%) & WAO-AHZ(\%) \\
\hline \multicolumn{7}{|c|}{ (a) Jiang-Shu test } \\
\hline 200 & 9.987 & +7.87 & +11.15 & 14.39 & +9.95 & +13.00 \\
\hline 400 & 38.45 & +9.25 & +11.93 & 57.16 & +10.07 & +12.84 \\
\hline 800 & 153 & +8.93 & +11.97 & 229.2 & +9.80 & +12.28 \\
\hline \multicolumn{7}{|c|}{ (b) Shu-Osher test with reconstruction along conservative variables } \\
\hline 200 & 3.06 & +10.30 & +17.29 & 4.094 & +11.13 & +18.70 \\
\hline 400 & 12.42 & +10.52 & +17.75 & 16.54 & +11.27 & +18.76 \\
\hline 800 & 49.09 & +9.89 & +15.64 & 66.79 & +10.22 & +17.56 \\
\hline \multicolumn{7}{|c|}{ (c) Lax test with reconstruction along characteristic variables } \\
\hline 200 & 3.108 & +10.15 & +16.04 & 10.82 & +9.61 & +11.67 \\
\hline 400 & 12.11 & +13.81 & +15.31 & 43 & +9.00 & +10.32 \\
\hline 800 & 47.92 & +13.16 & +19.70 & 172.2 & +9.22 & +9.93 \\
\hline \multicolumn{7}{|c|}{ (d) Spherical Sod test with reconstruction along conservative variables } \\
\hline 200 & 4.352 & +14.14 & +17.32 & 5.332 & +11.52 & +19.70 \\
\hline 400 & 17.05 & +9.16 & +15.88 & 21.21 & +10.62 & +18.67 \\
\hline 800 & 65.22 & +12.74 & +18.72 & 84.66 & +10.78 & +18.36 \\
\hline
\end{tabular}

reconstructions is not needed. This makes the CWENOZ-AO scheme more efficient in terms of computational cost.

In order to compare the computational efficiency of the adaptive order reconstructions, we compiled claw1dArena in release mode, which corresponds to - 03 optimization level of the $\mathrm{Gnu} \mathrm{C}++$ compiler. We measured the computational time required by each scheme to solve the numerical experiments proposed in the previous sections, by using three grids, precisely $N=200,400,800$ cells, and a CFL of 0.45 . We repeated this step five times and then we computed the median of the CPU times. Table 4 contains the results obtained on a quadcore Intel Core i7-6600U with clock speed $2.60 \mathrm{GHz}$ (left) and on a dualcore Intel Core i3-2100T with clock speed 2.50GHz (right).

Table 4 shows the CPU times in seconds for the CWENOZ-AO reconstruction. Instead, for the WENO-AO schemes we provide the difference in percentage compared to the time required by the CWENOZ-AO scheme. The CPU times for the gas-dynamics problems of ShuOsher and spherical Sod are computed without employing reconstruction along characteristic variables. Instead, the CPU times for the Lax test are computed by using reconstruction along characteristic variables. We observe that, as we expected, although the accuracy of the schemes is comparable, the major difference is given by their computational cost. In fact, both WENO-AO reconstructions require a larger computational time and therefore there is an increasing advantage in using the CWENOZ-AO type reconstruction.

\section{Conclusion}

In this paper we have presented a novel approach to adaptive order essentially non-oscillatory reconstruction. Our technique relies on the CWENOZ reconstruction with an optimal polyno- 
mial of degree $G$ and, in order to preserve the optimal accuracy on smooth data, the candidate polynomials are split into two families. The first family includes all polynomials with degree at least $G / 2$ and these are given $\mathcal{O}(1)$ linear weights, as in the usual weighted essentially nonoscillatory reconstructions. The second family is composed of those candidate polynomials with degree lower than $G / 2$, which would lower the accuracy on smooth data if given $\mathcal{O}(1)$ linear weights. These latter are thus associated to infinitesimal linear weights, proportional to $\Delta x^{r}$ with $r$ larger than the gap between $G / 2$ and the degree of the polynomial.

The main result of this paper is the analysis of the reconstruction, which gives sufficient conditions on the reconstruction parameter that guarantee the optimal convergence rates on smooth data.

As an application we have constructed and tested a CWZ753 that mimics the accuracy of WENO-AO $(7,5,3)$ reconstructions already present in the literature. The novel reconstruction shows similar accuracy to WENO-AO $(7,5,3)$ of $[2,5]$ on smooth data and, in some numerical tests, slightly reduces the onset of the spurious oscillations. Since our approach computes the set of nonlinear weights in one go, without resorting to iterative or hierarchic constructions, interesting savings in computational time could be demonstrated.

We point out that Proposition 2, as its analogous result in [16], is valid independently on the type of grid and number of space dimensions. Therefore, any choice of $\tau$ as in (10) can be employed to define CWENOZ-AO in much more general situations than the ones described in this paper. In particular, we expect that the present approach can yield very fast and accurate reconstructions in more than one space dimensions, since its CPU time savings could be combined with the avoidance of dimensional splitting that is made possible by the CWENOZ approach.

Even though we have considered the finite volume formulation, we believe that the same results extend straightforwardly to the finite difference case. Furthermore, also the recently proposed Multiresolution WENO schemes [49,50], which are based on a hierarchical computation for the nonlinear weights, might be amenable to be sped up following the ideas of this paper.

\section{References}

1. Aràndiga, F., Baeza, A., Belda, A.M., Mulet, P.: Analysis of WENO schemes for full and global accuracy. SIAM J. Numer. Anal. 49(2), 893-915 (2011). https://doi.org/10.1137/100791579

2. Arbogast, T., Huang, C.S., Zhao, X.: Accuracy of WENO and adaptive order WENO reconstructions for solving conservation laws. SIAM J. Numer. Anal. 56(3), 1818-1947 (2018). https://doi.org/10.1137/ $17 \mathrm{M} 1154758$

3. Baeza, A., Bürger, R., Mulet, P., Zorío, D.: Central WENO schemes through a global average weight. J. Sci. Comput. 78(1), 499-530 (2019). https://doi.org/10.1007/s10915-018-0773-z

4. Balsara, D.S., Garain, S., Florinski, V., Boscheri, W.: An efficient class of WENO schemes with adaptive order for unstructured meshes. J. Comput. Phys. 404, 109062 (2020). https://doi.org/10.1016/j.jcp.2019. 109062

5. Balsara, D.S., Garain, S., Shu, C.W.: An efficient class of WENO schemes with adaptive order. J. Comput. Phys. 326, 780-804 (2016). https://doi.org/10.1016/j.jcp.2016.09.009

6. Borges, R., Carmona, M., Costa, B., Don, W.S.: An improved weighted essentially non-oscillatory scheme for hyperbolic conservation laws. J. Comput. Phys. 227, 3191-3211 (2008). https://doi.org/10.1016/j.jcp. 2007.11.038

7. Boscheri, W., Balsara, D.S.: High order direct Arbitrary-Lagrangian-Eulerian (ALE) $\mathrm{P}_{\mathrm{N}} \mathrm{P}_{\mathrm{M}}$ schemes with WENO Adaptive-Order reconstruction on unstructured meshes. J. Comput. Phys. 398, 108899 (2019). https://doi.org/10.1016/j.jcp.2019.108899

8. Butcher, J.C.: Numerical Methods for Ordinary Differential Equations, 2nd edn. Wiley, NY (2008)

9. Capdeville, G.: A central WENO scheme for solving hyperbolic conservation laws on non-uniform meshes. J. Comput. Phys. 227, 2977-3014 (2008). https://doi.org/10.1016/j.jcp.2007.11.029 
10. Capdeville, G.: A high-order multi-dimensional HLL-Riemann solver for non-linear Euler equations. J. Comput. Phys. 230(8), 2915-2951 (2011). https://doi.org/10.1016/j.jcp.2010.12.043

11. Castro, M., Costa, B., Don, W.S.: High order weighted essentially non-oscillatory WENO-Z schemes for hyperbolic conservation laws. J. Comput. Phys. 230(5), 1766-1792 (2011). https://doi.org/10.1016/j.jcp. 2010.11.028

12. Castro-Dìaz, M.J., Semplice, M.: Third- and fourth-order well-balanced schemes for the shallow water equations based on the CWENO reconstruction. Int. J. Numer. Methods Fluid 89(8), 304-325 (2019). https://doi.org/10.1002/fld.4700

13. Cravero, I., Puppo, G., Semplice, M., Visconti, G.: Cool WENO schemes Comput. Fluids 169, 71-86 (2018). https://doi.org/10.1016/j.compfluid.2017.07.022

14. Cravero, I., Puppo, G., Semplice, M., Visconti, G.: CWENO: uniformly accurate reconstructions for balance laws. Math. Comput. 87(312), 1689-1719 (2018). https://doi.org/10.1090/mcom/3273

15. Cravero, I., Semplice, M.: On the accuracy of WENO and CWENO reconstructions of third order on nonuniform meshes. J. Scientific Comput. 67, 1219-1246 (2016). https://doi.org/10.1007/s10915-0150123-3

16. Cravero, I., Semplice, M., Visconti, G.: Optimal definition of the nonlinear weights in multidimensional Central WENOZ reconstructions. SIAM J. Numer. Anal. 57(5), 2328-2358 (2019). https://doi.org/10. 1007/s10915-015-0123-3

17. Don, W.S., Borges, R.: Accuracy of the weighted essentially non-oscillatory conservative finite difference schemes. J. Comput. Phys. 250, 347-372 (2013). https://doi.org/10.1016/j.jcp.2013.05.018

18. Dumbser, M., Boscheri, W., Semplice, M.: Central WENO subcell finite volume limiters for ADER Discontinuous Galerkin schemes on fixed and moving unstructured meshes. Commun. Comput. Phys. 25(2), 311-346 (2019). https://doi.org/10.4208/cicp.OA-2018-0069

19. Dumbser, M., Boscheri, W., Semplice, M., Russo, G.: Central weighted ENO schemes for hyperbolic conservation laws on fixed and moving unstructered meshes. SIAM J. Sci. Comput. 39(6), A2564-A2591 (2017). https://doi.org/10.1137/17M1111036

20. Dumbser, M., Käser, M.: Arbitrary high order non-oscillatory finite volume schemes on unstructured meshes for linear hyperbolic systems. J. Comput. Phys. 221(2), 693-723 (2007). https://doi.org/10.1016/ j.jcp.2006.06.043

21. Falcone, M., Paolucci, G., Tozza, S.: Multidimensional smoothness indicators for first-order HamiltonJacobi equations. J. Comput. Phys. (2020). https://doi.org/10.1016/j.jcp.2020.109360

22. Gerster, S., Herty, M.: Entropies and symmetrization of hyperbolic stochastic Galerkin formulations. Commun. Comput. Phys. 27(3), 639-671 (2020). https://doi.org/10.4208/cicp.OA-2019-0047

23. Ha, Y., Ho Kim, C., Ju Lee, Y., Yoon, J.: An improved weighted essentially non-oscillatory scheme with a new smoothness indicator. J. Comput. Phys. 232(1), 68-86 (2013). https://doi.org/10.1016/j.jcp.2012. 06.016

24. Harten, A., Engquist, B., Osher, S., Chakravarthy, S.: Uniformly high order accurate essentially non-oscillatory schemes, III. J. Comput. Phys. 71(2), 231-303 (1987). https://doi.org/10.1016/00219991(87)90031-3

25. Henrick, A.K., Aslam, T.D., Powers, J.M.: Mapped weighted essentially non-oscillatory schemes: achieving optimal order near critical points. J. Comput. Phys. 207, 542-567 (2005). https://doi.org/10.1016/j. jcp.2005.01.023

26. $\mathrm{Hu}, \mathrm{C}$. , Shu, C.W.: Weighted essentially non-oscillatory schemes on triangular meshes. J. Comput. Phys. 150(1), 97-127 (1999). https://doi.org/10.1006/jcph.1998.6165

27. Jiang, G.S., Peng, D.: Weighted ENO schemes for Hamilton-Jacobi equations. SIAM J. Sci. Comput. 21, 2126-2143 (2000)

28. Jiang, G.S., Shu, C.W.: Efficient implementation of weighted ENO schemes. J. Comput. Phys. 126, 202-228 (1996)

29. Kolb, O.: On the full and global accuracy of a compact third order WENO scheme. SIAM J. Numer. Anal. 52(5), 2335-2355 (2014). https://doi.org/10.1137/130947568

30. Kumar, R., Chandrashekar, P.: Simple smoothness indicator and multi-level adaptive order WENO scheme for hyperbolic conservation laws. J. Comput. Phys. 375, 1059-1090 (2018). https://doi.org/10.1016/j.jcp. 2018.09.027

31. Lahooti, M., Pishevar, A.: A new fourth order central WENO method for $3 \mathrm{D}$ hyperbolic conservation laws. Appl. Math. Comput. 218(20), 10258-10270 (2012). https://doi.org/10.1016/j.amc.2012.04.003

32. Levy, D., Puppo, G., Russo, G.: Compact central WENO schemes for multidimensional conservation laws. SIAM J. Sci. Comput. 22(2), 656-672 (2000). https://doi.org/10.1137/S1064827599359461

33. Naumann, A., Kolb, O., Semplice, M.: On a third order CWENO boundary treatment with application to networks of hyperbolic conservation laws. Appl. Math. Comput. 325, 252-270 (2018). https://doi.org/ 10.1016/j.amc.2017.12.041 
34. Puppo, G.: Numerical entropy production for central schemes. SIAM J. Sci. Comput. 25(4), 1382-1415 (2003). https://doi.org/10.1137/S1064827502386712

35. Puppo, G., Semplice, M.: Numerical entropy and adaptivity for finite volume schemes. Commun. Comput. Phys. 10(5), 1132-1160 (2011). https://doi.org/10.4208/cicp.250909.210111a

36. Qiu, J., Shu, C.W.: On the construction, comparison, and local characteristic decomposition for high-order central WENO schemes. J. Comput. Phys. 183(1), 187-209 (2002). https://doi.org/10.1006/jcph.2002. 7191

37. Semplice, M., Coco, A., Russo, G.: Adaptive mesh refinement for hyperbolic systems based on third-order compact WENO reconstruction. J. Sci. Comput. 66, 692-724 (2016). https://doi.org/10.1007/s10915015-0038-z

38. Semplice, M., Visconti, G.: claw1dArena v1.1 (2020). https://doi.org/10.5281/zenodo.2641724

39. Shi, J., Hu, C., Shu, C.W.: A technique of treating negative weights in WENO schemes. J. Comput. Phys. 175(1), 108-127 (2002). https://doi.org/10.1006/jcph.2001.6892

40. Shu, C.W.: Essentially non-oscillatory and weighted essentially non-oscillatory schemes for hyperbolic conservation laws. NASA/CR-97-206253 ICASE Report No.97-65 (1997)

41. Shu, C.W.: Essentially non-oscillatory and weighted essentially non-oscillatory schemes for hyperbolic conservation laws. Advanced numerical approximation of nonlinear hyperbolic equations (Cetraro, 1997). Lecture Notes in Math, vol. 1697, pp. 325-432. Springer, Berlin (1998)

42. Shu, C.W., Osher, S.: Efficient implementation of essentially non-oscillatory shock-capturing schemes II. J. Comput. Phys. 83, 32-78 (1989)

43. Toro, E.F.: Riemann Solvers and Numerical Methods for Fluid Dynamics, 3rd edn. Springer, Berlin (2009)

44. Zheng, F., Shu, C.W., Qiu, J.: High order finite difference Hermite WENO schemes for the HamiltonJacobi equations on unstructured meshes. Comput. Fluids 183, 53-65 (2019). https://doi.org/10.1016/j. compfluid.2019.02.010

45. Zhou, J., Cai, L., Zhou, F.Q.: New high-resolution scheme for three-dimensional nonlinear hyperbolic conservation laws. Appl. Math. Comput. 198(2), 770-786 (2008). https://doi.org/10.1016/j.amc.2007. 09.017

46. Zhu, J., Qiu, J.: A new third order finite volume weighted essentially non-oscillatory scheme on tetrahedral meshes. J. Comput. Phys. 349, 220-232 (2017). https://doi.org/10.1016/j.jcp.2017.08.021

47. Zhu, J., Qiu, J.: A new type of finite volume WENO schemes for hyperbolic conservation laws. J. Sci. Comput. 73, 1338-1359 (2017). https://doi.org/10.1007/s10915-017-0486-8

48. Zhu, J., Qiu, J.: New finite volume weighted essentially nonoscillatory schemes on triangular meshes. SIAM J. Sci. Comput. 40(2), A903-A928 (2018). https://doi.org/10.1137/17M1112790

49. Zhu, J., Shu, C.W.: A new type of multi-resolution WENO schemes with increasingly higher order of accuracy. J. Comput. Phys. 375, 659-683 (2018). https://doi.org/10.1016/j.jcp.2018.09.003

50. Zhu, J., Shu, C.W.: A new type of multi-resolution WENO schemes with increasingly higher order of accuracy on triangular meshes. J. Comput. Phys. 392, 19-33 (2019). https://doi.org/10.1016/j.jcp.2019. 04.027

Publisher's Note Springer Nature remains neutral with regard to jurisdictional claims in published maps and institutional affiliations. 